\title{
3 Das Jenseits des Totenreichs: SHADOW OF THE colossus
}

\section{Die orpheische Spur}

Es ist eine der ältesten Geschichten, die wir kennen: dass einer den Tod eines geliebten Menschen nicht erträgt und darum selbst auf Tuchfühlung mit dem Jenseits geht. Von Orpheus, der versucht, Eurydike der Unterwelt abzutrotzen, über Dante, der in den tiefsten Höllenkreis hinabsteigen und den Läuterungsberg erklimmen muss, ehe ihm im Paradies ein Wiedersehen mit Beatrice gewährt wird, bis hin zur zeitgenössischen Fantasy. Hier findet sich das Motiv etwa bei David Gemmell, wenn Druss sich entscheidet, das Tal der Toten zu bereisen, um Rowena die Rückkehr ins Leben zu ermöglichen. ${ }^{1}$ Ganz allgemein zählt jene Öffnung auf die Welt der Toten hin, die wechselseitige Durchdringung der vermeintlich geschiedenen Seinssphären, zu den unverzichtbaren Topoi der - in einem weiteren Sinn - fantastischen Populärkultur: Kein Twilight, kein Harry Potter, kein Game of Thrones kommt ohne sie aus. ${ }^{2}$ Und noch in einer Superhelden-Interpretation wie der Netflix-Serie THE PUNISHER (USA 2017-2019; Entwicklung: Steve Lightfoot), die auf schmerzliche Weise von den Verwerfungen der Gegenwart gezeichnet ist, erklingt gleichsam ein Echo des orpheischen Gesangs, da es mit Frank Castle (Jon Bernthal) schließlich so weit kommt, dass er, nun selbst ein blutender, gequälter, sterbender Körper, eine Wahl zu treffen hat, ob er mit seiner ermordeten Frau im schwarzen Liebesnichts verbleiben oder in eine Welt des Leidens zurückkehren möchte. ${ }^{3}$

Kein Wunder also, dass Hans Richard Brittnacher das Motiv der Jenseitsreise „zum Urgestein der literarischen und bildkünstlerischen Phantastik“ zählt. ${ }^{4}$ Mit ihm werde

1 Vgl. David Gemmell: The First Chronicles of Druss the Legend. New York 1994, S. 281-292.

2 Wohlgemerkt rede ich hier von den Medienverbünden, die unter den genannten Markennamen firmieren; neben Kinofilmen (bzw. einer Fernsehserie) und den Romanen, auf denen sie basieren, umfassen selbige Video- und Brettspiele, Comics, Fanfiction verschiedener Art, diverse Spin-Offs, Poster, Kalender, Tassen und allerlei sonstige Paraphernalien.

3 Vgl. Staffel 1, Episode 12: Home. Vielleicht wäre es interessant, die Figur Frank Castle, wie Lightfoot sie deutet, von ihrer Anlage her als eine Art umgekehrten Orpheus zu verstehen, der Verlust und Schuld nur ertragen kann, indem er die Metapher von der „Hölle des Krieges“ als Handlungsmatrix begreift, um die ganze Welt in eine ebensolche Hölle zu verwandeln.

4 Hans Richard Brittnacher: Jenseitsreisen. Der Traum und Alptraum vom Leben nach dem Tod. In: Christine Lötscher/Petra Schrackmann/Ingrid Tomkowiak/Aleta-Amirée von Holzen (Hg.): Übergänge und Entgrenzungen in der Fantastik. Münster 2014, S. 57-70, hier: S. 57. 
ein Terrain abgesteckt, auf dem sich die Produktivkräfte des Phantastischen entwickeln und bewähren konnten: in einer exzessiven Imagination, die es sich erlauben durfte, die üblichen Einsprüche der Empirie abzustreifen; in einem ungenierten Sprechen über Tabus, auch die letzten; und schließlich im Entschluss, einen Blick ins Herz der Finsternis zu werfen und die Schreckstarre erzählend zu verflüssigen. ${ }^{5}$

Auch SHADOW OF THE COLOSSUS ist letztlich ein orpheisches Lied. Seit seinem ersten Erscheinen im Herbst $2005^{6}$ wird das unter der Leitung Fumito Uedas von Team ICO entwickelte Videospiel als „Meisterwerk“ gehandelt. ${ }^{7}$ Tatsächlich zählt es zu den nicht eben zahlreichen Spielen, die als solche - also nicht nur als Exempel, an denen die Möglichkeiten und Grenzen des Mediums diskutiert werden - eine eingehendere wissenschaftliche Betrachtung erfahren haben. Die so entstandenen Beiträge belegen, dass es durchaus fruchtbar sein kann, Videospiele als künstlerische Werke ernst zu nehmen. Als solche ermöglichen sie eine ästhetische Erfahrung, die ohne sie schlechterdings nicht zu haben ist. ${ }^{8}$

Vor dem Hintergrund dieser Diskussion gilt es, die Perspektive auf SHADOW OF THE CoLOSSUS nicht über Gebühr zu verengen. Wenn ich im Folgenden untersuche, wie sich das Spiel zum Fantasy-Modus und dessen Permutationen verhält, will ich also zugleich die orpheische Spur im Auge behalten. Dies nicht, um SHADOW OF THE COLOSSUS von einer anderen Seite her in ein vorgegebenes Deutungsschema einzuzwängen oder das Spiel unter einem kulturhistorischen Trümmerhaufen zu begraben. Sondern gewissermaßen als Gegengewicht zu den im ersten Teil dieser Studie entwickelten Thesen; als Maßnahme mithin, die der Würdigung des Widerständigen und Rätselhaften an SHADOW OF THE COLOSSUS dient.

Wer reist hier ins Jenseits, und zu welchem Zweck? Was hat es überhaupt mit dem Jenseits auf sich? Ist es ein infernalischer Raum, soll es eine Himmelsahnung vermitteln, oder ähnelt es eher einem antiken Schattenreich? Weiterhin:

5 Brittnacher: Jenseitsreisen, S. 57.

6 Bluepoint Games entwickelte ein HD-Remaster von SHADOW OF THE COLOSSUS, welches das ursprünglich für die Playstation 2 konzipierte Spiel gemäß den Standards der folgenden Konsolengeneration aktualisierte und 2011 veröffentlicht wurde; 2018 folgte dann ein einhellig gelobtes Playstation-4-Remake, für das ebenfalls Bluepoint Games verantwortlich zeichnete. Letzteres stand mir während der Arbeit an dieser Analyse zur Verfügung.

7 Vgl. z. B. Christian Huberts: Raumtemperatur. Marshall McLuhans Kategorien „heiß“ und „kalt“ im Computerspiel. Salzhemmendorf 2010, S. 57.

8 Vgl. Martin Feige: Kunst, S. 186f.; vgl. auch: Damien Mecheri: The Works of Fumito Ueda. A Different Perspective on Video Games. Toulouse 2019. In seinem Buch entwickelt Mecheri eine Auteur-Perspektive auf Ueda. Er versucht u. a. zu bestimmen, inwieweit dieser beim Game Design einen distinkten Stil entwickelt hat; dabei stellt er auch die Frage, ob Uedas Spiele als Kunst gelten können. 
Welche Finsternis wird, um mit Brittnacher zu reden, in den Blick genommen, und gibt es eine Starre, die sich erzählend verflüssigt? Schließlich: Wenn es zutreffen sollte, dass das Medium Videospiel bezogen auf jene Art von Fantasy, die hier in Rede steht, eine privilegierte Position einnimmt - was bedeutet das hinsichtlich der Poetik von SHADOW OF THE COLOSSUS?

Ich will mit der einleitenden Filmsequenz des Spieles beginnen und dabei versuchen, mich langsam einer Antwort auf die genannten Fragen anzunähern.

\section{Melancholische Erhabenheit}

Ein paar Sekunden verweilt die Kamera auf dem vollen, von Wolken umlagerten Mond, dann macht sie sich daran, dem Flug eines Adlers zu folgen, schwenkt schräg nach unten, gleitet gemeinsam mit dem Tier hinab in eine zerklüftete, von Dunst und Nebel durchwaberte Berglandschaft. Vorbei an einem Wasserfall geht es, entlang der schroffen, steil aufragenden Wände einer Schlucht, die so hoch sich türmen, dass der Nachthimmel darüber nur noch erahnt werden kann. Dann, als der Adler einen schmalen Pfad erreicht, der auf den ersten Blick kaum von dem Felsen zu unterscheiden ist, bleibt die Kamera zurück. Sie lässt den Vogel seinen Flug fortsetzen, wohin auch immer, und schließt sich nun einem einsamen Reiter an, der einem gleichfalls unbekannten Ziel entgegenstrebt.

Zunächst sehen wir den Reiter nur von hinten und seitlich aus einiger Distanz. Er scheint sehr jung, trägt einen Umhang. An seiner Hüfte hängt ein Schwert und vor ihm, auf dem Rücken des schwarzen Pferdes, befindet sich, halb sitzend, halb liegend, eine leblose, in eine Decke oder einen Mantel gehüllte Frau. Das Erste, was wir über den Reiter erfahren, ist, dass eine unbedingte Entschlossenheit ihn antreibt. Als er an eine Stelle kommt, wo der Pfad eingebrochen ist, und ein klaffender Riss in schier bodenlose Tiefe führt, zwingt er sein widerstrebendes Pferd, den Sprung zu wagen. Es gelingt, und unbeirrt setzt der Reiter seine Reise fort. Bald führt ihn sein Weg durch einen Wald, den der Mond in zauberisch-verwunschenes Licht taucht, und vorbei an einem Fluss. Ein Gewitter bricht aus, und der Reiter lässt sein Pferd unter einem Felsvorsprung rasten.

Schließlich kommt er zu einer gewaltigen Ruine, die längst schon von Bäumen, Sträuchern, Kletterpflanzen und Gestrüpp in Beschlag genommen wurde. Handelt es sich um einen Tempel, eine Festung, ein Bollwerk? Jedenfalls verliert sich die Mauerflucht im Wald; schier unmöglich scheint es, das Gebäude zu umrunden. Doch zwischen zwei Türmen tut sich eine Öffnung auf. Den Spielerinnen und Spielern bleibt keine Zeit, sich zu fragen, was es mit der Ruine auf sich haben mag, denn schon setzt der Reiter seinen Weg fort. Durch jene Öffnung hindurch geht es, und während die Kamera, zunächst in Untersicht, zwischen den 
Mauern hindurch in den Himmel blickt, ein blassgoldenes Licht durch die Schatten schneidet, schwingt sich die bislang eher zurückhaltende Musik zu sakralem Pathos auf. Nun findet die Kamera ihren Platz hinter dem Reiter und seinem Pferd, blickt über ihre Schulter, während die enge Öffnung sich weitet, die Chöre und Streicher begleitend dazu immer weiter ansteigen, und es folgt ein Moment, in dessen Bildlichkeit die enigmatische Größe von SHADOW OF THE COLOSSUS zu einem Emblem der Melancholie und Erhabenheit sich verfestigt.

Vor den Augen der Spielerinnen und Spieler tut sich ein horizontweites, von einer kargen Wüste durchzogenes und von Felsen umstandenes Tal auf; eine Brücke überspannt dieses Tal, führt pfeilgerade auf ein weiteres gewaltiges Gebäude zu, das, seiner riesenhaften Größe zum Trotz, nur als Silhouette in der Ferne erkennbar ist. Die Kamera dreht sich nach oben, hin zu dem gleichzeitig wolkigen und sonnendurchglühten Himmel. Dann, nach einem Schnitt, werden die wahren Ausmaße der Szenerie deutlich: Nun entfernt sich die Kamera von Reiter und Pferd, die langsam beginnen, die Brücke zu überqueren. Immer weiter entfernt sie sich, und je größer die Distanz zu jenen zuerst kleinen, dann winzigen Gestalten wird (die Frau ist schon überhaupt nicht mehr zu erkennen), desto unfasslicher erscheinen sie: die Weite und Leere der Welt, die der Spieler soeben betreten hat, die Macht und das Alter der halbverfallenen Bogenbrücke - deren Bögen übrigens, ein bemerkenswertes Detail, genau genommen Halbbögen sind, die wie überdimensionierte Wegweiser immer in eine Richtung zeigen: in das Tal hinein, weg von den Landen, die der Reiter bislang bereist hat; als wollte die Brücke selbst deutlich machen, dass es für denjenigen, der einmal den Fuß auf sie gesetzt hat, keine Rückkehr mehr geben kann.

Spätestens an dieser Stelle verschiebt sich die rätselnde Neugier der Spielerinnen und Spieler: Mag sie bislang dem Reiter und der Frau gegolten haben, richtet sie sich nun - eine Verlagerung, die beim Anblick der ersten Ruine ihren Ausgang genommen hat - auf die Welt selbst. Die dringendsten Fragen sind also nicht mehr: Wer ist der Reiter? Was verbindet ihn mit seiner vielleicht toten, jedenfalls in tiefer Ohnmacht gefangenen Gefährtin? Und wohin führt ihn sein Ritt? Ist er etwa auf der Flucht? Sondern: Was ist das für eine Welt? Wer hat diese gewaltigen Bauwerke errichtet? Wann ist das geschehen? Und warum stehen sie mitten in einer menschenleeren Einöde?

Anstatt diese Fragen zu beantworten, vollzieht das Spiel eine Bewegung, mit der es gleichsam auf sich selbst zurückweist: Die Musik verklingt, nur noch das Rauschen des Windes ist zu hören, einmal mehr schwenkt die Kamera in den Himmel, und nach einer Weißblende erscheint der Titelschriftzug zwischen regenschweren Wolken, verbunden mit der Aufforderung, einen Knopf zu drücken. Man mag in dieser Inszenierung durchaus eine Geste erkennen, mittels derer SHADOW OF THE COLOSSUS die eigene Hermetik unterstreicht und zugleich 
die Bereitschaft herausstellt, die Erwartungen der Spielerinnen und Spieler zu frustrieren. Dafür spricht, dass die restlichen zehn Minuten der Eröffnungssequenz, während sie vorgeblich manche Frage beantworten, eigentlich immer nur neue Rätsel aufwerfen.

Weiter geht es mit einem Adler (vermutlich demselben, den wir bereits ganz zu Beginn kennenlernten); gemeinsam mit ihm umfliegt die Kamera das Gebäude am jenseitigen Ende der Brücke, kommt vor einem großen Steintor zum Stehen, auf das der Reiter unbeirrt zuhält. Wie von Geisterhand hebt sich dieses Tor. Erneut widerstrebt das Pferd; der Reiter treibt es an, die Öffnung zu durchqueren und die dahinter liegende Treppe hinabzuschreiten. Kaum sind die ersten Stufen genommen, da senkt sich das Tor, wie es sich zuvor gehoben hat: von einer unsichtbaren Kraft getrieben und mit lautem Knirschen. Etwas Endgültiges liegt in dieser Bewegung, eine Schicksalsschwere, die verstärkt wird von der Stille - noch immer ist keine Musik zu hören -, in der die Hufschläge des Pferdes hallen, als es einem Rundweg folgt, der entlang der Mauern des in seinen Ausmaßen wiederum kaum fasslichen, kuppelartigen Gewölbes und hinab in die Tiefe führt; nur erhellt vom Licht, das durch die Öffnung der Kuppel hereinströmt.

Nun endlich ist der Reiter am Ziel angelangt. Er lenkt das Pferd in eine langgezogene Halle, die von Nischen gesäumt ist, in denen steinerne Idole aufragen; am Ende der Halle findet sich ein Altar; offene Bogenfenster lassen die einsame Weite erahnen, die dahinter liegt, und auch die Halle wird zusätzlich beleuchtet vom Licht der Sonne, das durch eine Öffnung im Kuppeldach einfällt. Als der Reiter diese Halle erreicht, erklingt von Neuem sakral anmutende Musik, und zum ersten Mal kann man deutlich sein Gesicht erkennen. Doch wenn sich die Spielerinnen und Spieler erhofft haben sollten, dass diese Enthüllung etwas klären würde, sehen sie sich enttäuscht: Ernst und ruhig, beinah ausdruckslos, sind die in der Tat sehr jungen Züge des Reiters; sie geben kein Geheimnis preis.

Daran ändert sich auch nichts, als der Reiter seine Gefährtin - spätestens jetzt wird es zur Gewissheit, dass sie tot ist - vom Rücken des Pferdes nimmt, zum Altar hinüberträgt und auf die Steinplatte legt. Erst, als er die Decke wegnimmt, in die sie gehüllt war, und auf sie hinabblickt, tritt Trauer in sein Gesicht.

In einem weiteren Einschub sieht man eine von Rauch und Funkenflug halb verborgene Karte (die von den Spielerinnen und Spielern später als Weltkarte aufrufbar ist), und während eine hölzerne, an eine Eule gemahnende Maske langsam in den Bildvordergrund schwebt, hebt eine Erzählstimme zu äußerst kryptischen Erklärungen an, denen man eigentlich nur entnehmen kann, dass das Land, in das sich der Reiter hineinbegeben hat - und wir mit ihm -, ein verbotenes Land ist; und dass es möglich sein soll, in den Grenzen dieses Landes die Toten wieder zum Leben zu erwecken. 
Zurück im Tempel, denn um einen solchen handelt es sich offenbar bei dem gewaltigen Gebäude, blicken die Spielerinnen und Spieler einen Moment lang in das ebenfalls sehr ruhige und sehr junge Gesicht der leblosen Frau. Dann erheben sich fünf schwarze, schattenhafte Gestalten aus dem Boden. Der Reiter dreht sich um, zieht sein Schwert, dessen Klinge von bläulichem Licht umstrahlt ist, und richtet es auf die Schatten - das alles, ohne dass ein Zeichen von Angst, Aufregung oder Kampfeslust in seinem Gesicht erkennbar wäre -, die daraufhin in Rauch verwehen. Nun schwenkt die Kamera zu der Öffnung im Kuppeldach empor, die von gleißendem Licht erfüllt scheint, obwohl man nach einem Schnitt einen grauschwarzen, blitzdurchzuckten Gewitterhimmel sieht. Das sind durchaus ominöse Auspizien. Und auch die Stimme, die nun erklingt, gleichsam aus dem Gleißen herausschallt, wirkt wenig vertrauenerweckend, ist sie doch aus mehreren verzerrten Männer- und Frauenstimmen zusammengesetzt.

Wie alle verbalen Äußerungen, die im Verlauf von SHADOW OF THE COLOSSUS zu hören sind, ist der folgende Dialog in einer Kunstsprache gehalten, die das Japanische mit romanischen Lauten verbindet, wodurch die Hermetik des Spieles wohl zusätzlich betont wird, da es unabdingbar ist, zum Verständnis auf Untertitel zurückzugreifen. ${ }^{9}$ Was ist es, das wir aus der Unterredung zwischen dem Reiter, er heißt übrigens Wander, ${ }^{10}$ und jener geheimnisvollen Stimme lernen?

Zunächst einmal, dass Wander auf der Suche nach einem Wesen ist, das an diesem Ort am Ende der Welt leben soll, und dem Macht über die Seelen der Toten zugesprochen wird. Der Name des Wesens ist Dormin, und die vielgestaltige Stimme bestätigt: Wir sind Dormin. Die Bitte des Reiters lautet erwartungsgemäß, dass Dormin die junge Frau zurück ins Leben holen möge; wobei strenggenommen von ihrer „Seele“ die Rede ist. Das anscheinend gottähnliche Wesen erläutert, es liege in seiner Macht, diese Bitte zu erfüllen; nur deshalb allerdings, weil sich Wander im Besitz eines besonderen Schwertes, des „Ancient Sword“, befinde. Zunächst aber müsse er die Aufgabe erfüllen, die Dormin ihm stellt: Es gilt, die sechzehn Idole zu zerstören, die in der Halle aufgereiht stehen. Zwar sei dies einem Sterblichen an sich nicht möglich, doch würden in dem Land, das den Tempel umgibt, sechzehn Kolosse hausen, die Inkarnationen der Idole darstellen. Wenn es Wander gelingt, die Kolosse zu besiegen, werden auch die Idole fallen, und die Tote - sie heißt Mono ${ }^{11}$ - kann ihre Seele zurückerhalten. Der junge Mann erklärt sich bereit, zu tun, was Dormin von ihm verlangt. Die Warnung des

9 Vgl. Sarah Cameron Loyd Grey: Dead Time. Aporias and Critical Videogaming. In: symplokē 17 (2009), H. 1-2, S. 231-246, hier: S. 235.

10 Was man allerdings nur aus den Paratexten von SHADOW OF THE COLOSSUS erfährt; im Abspann etwa werden die Figuren namentlich genannt.

11 Auch dieser Name wird im Spiel selbst nicht genannt. 
Wesens, dass er womöglich einen wahrlich hohen Preis werde zahlen müssen, schlägt er in den Wind; das sei gleichgültig: „It doesn’t matter.“

Sowie Wander, noch immer mit unbewegtem Gesicht, diese Worte ausgesprochen hat, ist der Pakt besiegelt. „Very well ...“, entgegnet Dormin, und die Kamera fährt vorbei an Wander, der sich seltsamerweise in diesem Moment umschaut, und der toten Mono, gleitet zwischen den Säulen der Bogenfenster hindurch ins Freie, öffnet somit den Blick auf das weite, leere Land, in dem die Kolosse warten. Eine Orgel tönt, noch einmal erheben sich die Chöre, noch einmal zieht es die Kamera in den Himmel, sodass die sinkende Sonne in den Blick kommt, die, von Wolken verborgen, über den Hügeln steht. Und nachdem Wander den Hinweis erhalten hat - die erste von zahlreichen, mitunter sehr rätselhaften Hilfestellungen, die Dormin gibt -, dass er die Kolosse finden könne, wenn er sein Schwert ins Licht halte und dorthin reite, wo sich die Lichtstrahlen sammeln, beginnt das eigentliche Spiel.

\section{Die Entstehung des Weltgefühls}

Ich habe die Eröffnungssequenz von SHADOw OF THE COLOSSUS in dieser Ausführlichkeit beschrieben, weil ich glaube, dass sich in ihr - und zwar nicht so sehr in dem, was geschieht, sondern auch und vor allem im Wie der Inszenierung die Poetik des Spiels verdichtet. Das ist nicht selbstverständlich, wird die cutscene doch gemeinhin definiert als „eine Zwischensequenz, oft im Sinne einer Filmsequenz, die dem Spieler ohne dessen interaktive Beteiligung die Spielhandlung vermittelt““. ${ }^{12}$ Was die Eröffnungssequenz, die hier in Rede steht, an Handlung „vermittelt“, ist recht bescheiden: Ein junger Mann bringt eine tote Frau in einen Tempel, bittet ein gottähnliches Wesen, die Tote zum Leben zu erwecken, und bekommt von diesem den Auftrag, sechzehn Kolosse zu töten. Dafür braucht es keine Viertelstunde; und auch keine seitenlange Schilderung.

Aber die sprachliche Beschreibung einer Filmsequenz ist eben keine Reproduktion objektiv gegebener Informationen - und eben darum letztlich redundant -, sondern der Versuch, einen gedanklichen und emotionalen Gehalt zu fassen, den der oder die Schreibende in der jeweiligen Sequenz gestaltet findet. ${ }^{13}$ Insofern dient sie dazu, eine analytische Perspektive zu eröffnen, die eindeutig über das hinausgeht, was in den audiovisuellen Bildern an sich zu

12 GamesCoop: Theorien des Computerspiels zur Einführung. Hamburg 2012, S. 207.

13 Vgl. hierzu die Diskussion von Hermann Kappelhoffs filmanalytischer Methode im zweiten Kapitel der vorliegenden Studie. 
sehen und $\mathrm{zu}$ hören ist, weshalb sie auch nicht beispielsweise durch einen Link auf die Filmsequenz selbst ersetzt werden kann.

Was ist dann der Gehalt der Eröffnung von SHADOw OF THE COLOSSUS? Ex negativo lässt sie sich dadurch bestimmen, dass sie weder der Illustration von Handlung dient, noch dazu, SHADOW OF THE COLOSSUS abwechslungsreicher oder lebendiger zu machen, oder in einer actionreichen, an die Standards von Hollywood-Blockbustern angelehnten Inszenierung die Figuren und die narrativen Konflikte zu dynamisieren. Stattdessen wird hier ein Gefühl für die Welt des Spieles in Szene gesetzt, das zugleich ein Spielgefühl ist. Dieses Gefühl mag sich im Verlauf der folgenden sechs oder acht Stunden, mithin der Zeit, bis der letzte Koloss fällt, zwar vertiefen und verfestigen, vielleicht über kleinere Brüche hinweg, wird jedoch nicht aufgehoben oder ersetzt - bis zum Ende, das freilich eine eigene Analyse erfordert.

Ein Weltgefühl also. Zu seiner Entstehung braucht es die Dauer der einleitenden Filmsequenz; jene fünfzehn Minuten, die hier ein Geschehen beansprucht, das von seinem Sachgehalt her auch in fünf oder drei Minuten erzählt werden könnte. Es braucht die Langsamkeit des Rittes, den fast gemächlichen Rhythmus der Hufschläge und des Wandels von Nacht zu Morgen zu Mittag, von einem Wetter zum anderen, weil diese Langsamkeit, dieser Rhythmus, die Zeit sind, in der sich eine Welt entfaltet, die im selben Maß verwunschene Schönheit und unaufhebbare Trauer erfüllen. Wobei sich Traurigkeit und Melancholie, auf eine narrativ zu keinem Zeitpunkt des Spieles eingeholte Weise, mit der Ahnung verbinden, dass es eine Vergangenheit gegeben haben mag, in der diese Berge, Wälder und Ebenen nicht so einsam und verlassen waren. Es sind die Ruinen, denen sich diese Ahnung einschreibt; die Ruinen und der Vogelflug. Denn der Schwung der Adlerflügel setzt der langsamen, dabei unbeirrbaren Geradlinigkeit des Rittes eine andere Bewegungsqualität entgegen, die in ihrer freien und fast spielerischen Ungebundenheit die Kamera mitreißt und es vorstellbar macht, dass die Welt, die hier spürbar wird, auch einem froheren Gesetz unterstehen könnte.

In den beschriebenen Attributen ist die Welt von SHADOW OF THE COLOSSUS beispielhaft für die gefallenen Welten der Fantasy. Zumindest, was die alles durchdringende Melancholie und Einsamkeit angeht, sind diese Attribute auch oft und treffend bestimmt worden. ${ }^{14}$ Dennoch lohnt es sich, bei der Frage zu verweilen,

14 So spricht Grey von einer „deserted, melancholic landscape“; Christopher Michael Yap beschreibt die Spielwelt als „a wide and desolate place“ und betont die „nigh-complete isolation“ des verbotenen Landes. Grey: Dead Time, S. 232; Christopher Michael Yap: Reflections on SHADOW OF THE COLOSSUS (PS2; Team ICO, 2005), Pt. 1. In: In Medias Resolution, 22. Juli 2015, 
wie die Welt des Spieles konstruiert ist. Und zwar aus zweierlei Gründen: zum einen, um die Verbindung zwischen SHADOW OF THE COLOSSUS und dem, was ich die orpheische Spur genannt habe, zu erhellen; zum anderen, weil die Entwickler um Fumito Ueda ihrem Werk eine recht eigentümliche Beziehung zwischen Spieler und Avatar installiert haben, die man am leichtesten versteht - und das wiederum ist für eine poetologische Analyse von SHADOW OF THE COLOSSUS unerlässlich -, wenn man sich ihr über die Weltkonstruktion annähert.

\section{Die Zeitlichkeit des Asphodeliengrunds}

Können wir also noch genauer bestimmen, was es mit dieser ebenso schönen wie trauervollen Welt auf sich hat? In Bezug auf SHADOW OF THE COLOSSUS von einer orpheischen Spur zu sprechen, macht offenkundig nur dann Sinn, wenn sich die Spielwelt als jenseitige Topografie fassen lässt. Geht man von der christlichen Eschatologie aus, ist dem zweifellos nicht so. ${ }^{15}$ Eine paradiesische Seligkeit sucht man hier vergebens. Ebenso wie infernalische Qualen in der Tradition mittelalterlicher Jenseitsreisen, bei denen ,in einer Mischung aus Sadismus und Pedanterie [...] mit buchhalterischer Gründlichkeit für jede Sünde, jedes Vergehen exquisite Schindereien ersonnen werden“. ${ }^{16}$ Und sicherlich durchläuft Wander keinen Prozess der Läuterung, wie ihn die Seelen im Purgatorium erfahren.

Sehr wohl allerdings schließt die Gestaltung der Welt von SHADOW OF THE coLossus an antike Vorstellungen des Jenseits an. Asphodeliengrund - so nannten die Griechen den Wohnsitz der Toten, die es weder auf die Insel der Seligen geschafft hatten, noch zum Aufenthalt im Tartaros verdammt worden waren. Dieser Ort war „öde und abwechslungslos und bot keinen gesellschaftlichen Umgang“; die Toten mussten hier „für ewig“ hausen, und zwar als Schatten „ohne Blut und Bewusstsein“. ${ }^{17}$

Wie man sich diese wenig einladende Lokalität genauer vorzustellen hat, berichtet Vergil, wenn er Aeneas gemeinsam mit der Sibylle in die Unterwelt schickt:

https://www.chris-yap.com/single-post/2015/07/22/Reflections-on-Shadow-of-the-Colossus-PS2Team-ICO-2005-Pt-1 [letzter Zugriff: 12.05.2020].

15 Was bei einem japanischen Spiel auch nicht weiter verwundert.

16 Brittnacher: Jenseitsreisen, S. 58. Wobei man vielleicht hinzufügen sollte, dass die Folterexzesse, die etwa Dante beschreibt, rein gar nichts mit einer heutigen Theologie der Hölle zu tun haben.

17 Michael Grant/John Hazel: Lexikon der antiken Mythen und Gestalten [1976]. München 2009, S. 177. 
Heimlich gingen sie da einher in einsamer Nacht durch die Schattenwelt und durch die verödeten Paläste des Dis und seiner trostlosen Reiche. Der Weg war wie bei mattem Mondschein im Zwielicht der Wälder, wenn Jupiter den Himmel verdunkelt und schwarze Nacht allen Dingen die Farbe nimmt. ${ }^{18}$

Eine gewisse atmosphärische Parallelität zu SHADOW OF THE COLOSSUS liegt auf der Hand. Allerdings sind auch die Unterschiede kaum zu übersehen. Das geht schon damit los, dass die Welt, die Wander auf der Suche nach den Kolossen durchstreift, zwar „keinen gesellschaftlichen Umgang bietet“ und eben darum auch in gewisser Weise „öde“ ist, mitnichten aber „abwechslungslos“. Im Gegenteil bietet sie eine beachtliche Vielfalt an Landschaften und Schauplätzen: Wüsten, Steppen, Wiesen, Haine und Wälder, Täler, Schluchten, Klippen, Seen und Strände, Höhlen und allerlei Ruinen - womit die Aufzählung noch nicht vollständig wäre -, weshalb man auch nicht behaupten kann, dass allen Dingen ihre Farbe genommen sei (sondern nur einigen); und abgesehen davon findet Wanders Abenteuer zum größten Teil am Tage statt, wenngleich bei mitunter ziemlich trübem und sturmverdunkeltem Wetter.

Ist es dennoch angemessen, das Verbotene Land von SHADOW OF THE COLOSSUS als Jenseits, genauer: als Asphodeliengrund und Totenreich, zu bezeichnen? Wenn ja, dann lässt sich diese Behauptung nicht unter Verweis auf die Repräsentationsebene des Spieles rechtfertigen. Was sonst aber könnte sie begründen?

Eine schlichte Antwort auf diese Frage lautet: die Spielerfahrung selbst. Nachdem Dormin den jugendlichen Helden in die Welt von SHADOW OF THE COLOSsus entlassen hat, gibt es für diesen strenggenommen nur zweierlei zu tun: Er muss die Kolosse suchen und bekämpfen. Wie gestaltet sich das? Die Suche nach den Kolossen geht zwangsläufig einher mit einem Sich-Verlieren. Man verliert sich in der monumentalen Weite und Leere, die ebenso bezaubernd wie öde ist; verliert sich in der Zeitlichkeit der Suche, in den langen Ritten durch das Verbotene Land, den langen Ritten, während derer nichts, oder doch fast nichts geschieht, und die aus diesem Grund leicht in ein ermattendes Rätseln - „a kind of puzzle“ - ausarten können, wenn man, von den Lichtstrahlen geleitet, die, in der Klinge des Schwertes reflektiert, vage den Weg weisen, den nächsten Koloss sich aufzuspüren müht: ${ }^{19}$ einen Gegner, der selbst wie verloren oder aufgelöst

18 Vergil: Aeneis. Prosaübertragung, Nachwort und Namensverzeichnis von Volker Ebersbach. Stuttgart 1982, S. 166.

19 „The Forbidden Land is perhaps one of the largest digital landscapes in the PS2-era, and there have been many a times where players have been utterly confounded by the inability to find a colossus in such a wide and desolate place. Thankfully, the sword also functions as a magical light compass which points the way to the next colossus, but as it is only a line-ofsight pointer, it can become easy to run headlong into a mountainside or straight off a cliff if 
scheint in der kargen und geheimnisvollen Endlosigkeit dieser Welt, sodass man sich mitunter fühlen mag, als suche man eine Nadel im Heuhaufen.

In Anbetracht der riesenhaften Größe der allermeisten Kolosse (die sich freilich erst offenbart, ein ums andere Mal, wenn man sie gefunden hat) mag der Vergleich ziemlich unangebracht wirken. Doch gerade darum geht es: Es ist die Absurdität, etwas suchen zu müssen, das an sich schon jegliches Maß sprengt (zumal die Maße einer gewöhnlichen Spielfigur), aber dennoch verzwergt in einer noch gewaltigeren Maßlosigkeit. Diese Absurdität mag dazu führen, dass sich die Spielerinnen und Spieler in ihren Empfindungen dem Verbotenen Land angleichen. Man sollte hier nicht von Langeweile sprechen; was in Rede steht, ist eher eine existenzielle Ausleerung und Verödung.

Erschwerend kommt hinzu, dass SHADOW OF THE COLOSSUS die wenigen Handlungsmöglichkeiten, die dem Spieler ansonsten gegeben sind, mit kleinen Widerhaken versieht. So ist kaum zu übersehen, dass den Interaktionen mit der Spielwelt fast ausnahmslos etwas Missbräuchliches anhaftet. Man kann harmlose Echsen töten, die dann kläglich verenden (im Fall der Kristallechsen ist es zudem möglich, sich mit den Schwänzen der Tiere zu stärken); man kann sich Fischen und Habichten anhängen, die einen dann durchs Wasser ziehen beziehungsweise durch die Luft tragen; man kann Schildkröten erschrecken, die sich dann angstvoll in ihrem Panzer verkriechen. All diese Tiere haben eindeutig nicht auf Wander gewartet und ziehen keinerlei Gewinn daraus, dass er in ihr Revier eindringt - was sie übrigens mit den Kolossen verbindet. Selbst die harmlose Unternehmung des Früchte-Essens entbehrt nicht dieses missbräuchlichen Einschlags; man muss die Früchte nämlich von den Bäumen schießen; freiwillig gibt das Verbotene Land nicht einmal seine gewöhnlichsten Schätze preis.

Das führt uns zum nächsten Punkt. Sich mit SHADOw OF THE COLOSSUS zu beschäftigen, ist ja auch deshalb in keinem gewöhnlichen Sinn langweilig, weil die Welt des Spieles in ihrer Weite, ihrer Leere, ihrer Kargheit und ihrer Blüte eine immense Faszination ausstrahlt. In gewisser Weise ist sie ein einziges Geheimnis, und kaum eine Spielerin, kaum ein Spieler wird sich dem Wunsch entziehen können, dieses Geheimnis zu lüften. Tatsächlich richtet SHADOW OF THE COLOSSUS überall in den Verbotenen Landen gleichsam lockende Finger auf. Wie oft geschieht es, dass man in der Ferne eine Hügelkette, einen Tempel, den Eingang einer Schlucht erspäht, und, von Entdeckerlust getrieben, die mühselige Suche nach dem nächsten Gegner unterbricht, um die Rätsel dieser Welt zu erkunden! Aber auch diese Freuden sind keineswegs ungetrübt. Denn die Welt des Spieles

one is not mindful of the environment. Searching out each colossus also constitutes a kind of puzzle.“ Yap: Reflections on SHADOW OF THE COLOSSUS. 
verschließt sich den Wünschen, die sie zuvor erweckt hat. Zwar kann man mit etwas Findigkeit auch entfernte Ecken des Verbotenen Landes erreichen und vermeintlich unzugängliche Orte (wie beispielsweise Dormins Tempel) erklettern; zwar kann man manch eine in verwunschenen Winkeln verborgene Ruine erkunden, und sich etwa darüber wundern, wer wohl die Feuer entzündet hat, die hier in Metallschalen brennen. Aber nichts von allem, was man tut, wird einem der Antwort auf die Fragen, welche SHADOW OF THE COLOSSUS so insistierend stellt, auch nur das kleinste Stückchen näherbringen. Das Spiel hütet seine Geheimnisse so eifersüchtig wie Smaug das Zwergengold. Es ist sozusagen nicht lesbar vorsätzlich, absichtsvoll und in aller Entschiedenheit. ${ }^{20}$

Das mischt noch der eigentlich friedlichen und (jedenfalls bezogen auf Wanders Zustand) segensreichen Tätigkeit, an den über die Welt verstreuten Schreinen $\mathrm{zu}$ beten, etwas Frustranes bei. $\mathrm{Zu}$ wem betet man hier eigentlich? Für wen oder was? Und wie kann es sein, dass die Gebete augenscheinlich erhört werden, und sich dennoch nicht das Geringste ändert? Auch auf diese Fragen gibt es keine Antwort.

Ist die Welt von SHADOW OF THE COLOSSUS also ein Totenreich? Ja, das ist sie. Wie gesagt: nicht so sehr auf der Ebene dessen, was das Spiel repräsentiert und erzählt. Sondern als die Erfahrung, die es gestaltet. Verwirrt und verloren, von gequältem Überdruss und ungestillter Sehnsucht erfüllt, mögen die Spielerinnen und Spieler durch diese Welt irren - die so viel mehr verspricht, als sie zu halten bereit ist, und doch immer wieder lockt und verzaubert mit der Ahnung, was möglich sein könnte -, wie die Schatten, die ihren Wohnsitz im Asphodeliengrund nehmen mussten.

20 „In Massively Multiplayer Online Roleplaying Games (MMORPGs) such as WORLD OF WARCRAFT, there are also ruins. They seem to metaphorically lament a lost history. The ruins in SHADOW do this as well and, due to the fact that there is absolutely no trace of habitation, go further to point to their ultimate inexplicability. The ancient civilization did not make SHADow's crumbling temples and bridges in order for us to interpret them, for players have no code by which to do so. In WORLD OF WARCRAFT, there are quests, characters, and documents to help players piece together an admittedly spotty account. In SHADOW OF THE COLOSSUS, the ruins remain mute; they are about the utter absence of history." Grey: Dead Time, S. 234.

Man könnte zum Vergleich auch Rollenspiele heranziehen, die mit dem Spielprinzip der offenen Welt arbeiten, etwa SKYRIM oder THE WITCHER 3: THE WILD HUNT (CD Project Red, 2015). Beide Spiele verlocken ebenfalls zu Erkundungen, die weitab der Pfade der Hauptgeschichte führen, belohnen diese Erkundungen aber auf vielfache Weise; vor allem eben auch mit kleinen Geschichten, die immer wieder Neues über die jeweiligen Welten und ihre Bewohner preisgeben. Vgl. das zweite Kapitel dieser Studie. 
Ein Jenseits ist das verbotene Land von SHADOW OF THE COLOSSUS ebenso sehr in Hinblick auf sein Medium; auf das also, was Videospiele für gewöhnlich strukturell, narrativ und dramaturgisch ausmacht.

Christian Stöcker beschreibt das wie folgt:

Dieses Spiel macht alles falsch. [...] Es gibt keine Waffen-Upgrades, keine Individualisierungsmöglichkeiten, keine Explosionen, keinen fetzigen Soundtrack, keine bunten Farben. Der Held hat keine sichtbaren Muskeln und macht keine lässigen Sprüche - genau genommen sagt er fast nie etwas. Und es gibt, nach vielen, manchmal durchaus mühevollen Stunden, nicht mal ein richtiges Happy End. ${ }^{21}$

Unter Bezugnahme auf diese Sätze stellt Thomas Hensel fest, SHADOW OF THE COLOSSUS sei, was die Narration und Dramaturgie angeht, „monoton, wenn nicht vakuös“ und führt aus:

Zu dem irritierenden Umstand, dass die genreübliche Klimax als „stetige Alternation von Plateauphasen und Steigerungssprüngen“ fehlt, passt ein weiterer Befund, hat es doch den Anschein, als gehe nach jeder Niederstreckung eines Kolosses das Spiel selbst gleichsam in die Knie und wolle von vorn beginnen: Unser Held, nach einem Kampf aufgeladen mit der Essenz des gefallenen Giganten, wird bewußtlos und erwacht jedes Mal wieder in jener Tempelhalle, von der das Ganze seinen Ausgang nahm, ohne dass er eine wie auch immer geartete höhere Stufe erklommen hätte - das Spiel wird gewissermaßen auf ,Null` gestellt. Die Schwarzblende, sprich der totale Bildentzug des Spieles, korrespondiert mit der keine Klimax kennenden A-Narrativität des Spiels. ${ }^{22}$

Was Hensel hier beschreibt, ist eine zyklische Zeitlichkeit, die in ihren gleichförmigen Wiederholungen fast schon einer Stillstellung der Zeit gleichkommt. Es ist mithin die Zeit des Totenreichs, welche die Schatten dazu verdammt, als verblassende Erinnerungen ihrer selbst in einer endlos sich reflektierenden Leere auszuharren: ohne Vergangenheit und Zukunft, ohne jegliche Entwicklungsmöglichkeit und Seinspotenzialität. SHADOW OF THE colOSSUS schließt Spieler und Avatar in eine solche Zeitlichkeit ein, und folgt man Stöckers Ausführungen, so liegt der Gedanke nahe, dass auch die Reduktion der Spielmechaniken, das minimalistische Design, nicht zuletzt der Inthronisierung der Zeit des Totenreichs dienen.

Dazu passt, dass es zu dem Jenseits von SHADOw OF THE Colossus kein Diesseits gibt. Zwar ist Wanders Eintritt in das Verbotene Land deutlich markiert, und

21 Christian Stöcker: Ein stilles Meisterwerk. In: Spiegel Online, 24. Februar 2006, http:// www.spiegel.de/netzwelt/web/spiel-shadow-of-the-colossus-ein-stilles-meisterwerk-a-402686. html [letzter Zugriff: 12.05.2020].

22 Thomas Hensel: Autodestruktionen | Autoikonoklasmen des Computerspiels. In: Navigationen 12 (2012), H. 2, S. 99-116, hier: S. 108. 
Fumito Uedas Inszenierung lässt keinen Zweifel daran, dass hier in der Tat eine Grenze überschritten wird. Doch von den ersten Momenten der Eröffnungssequenz an erhält die Spielwelt ihr atmosphärisches Gepräge. Das damit einhergehende Gefühl von melancholischer Erhabenheit und allumfassender Einsamkeit mag sich späterhin verdichten oder intensivieren. Diese Veränderungen betreffen aber, wie gesagt, nicht den Kern der Spielerfahrung.

Schließlich sei noch einmal darauf hingewiesen, dass das Verbotene Land unter einer sinkenden Sonne steht. Auch diesbezüglich ist die Zeit stillgestellt, denn ganz gleich, wie viele Stunden man mit Wander durch die Wüsten und Wälder, über die Ebenen und Wiesen reitet, das Licht kennt weder Wechsel noch Wandel; oder treffender: Seine Dynamik ist, ebenso wie diejenige des Wetters, nicht temporal, sondern topografisch definiert, hängt also davon ab, welchen Winkel der Spielwelt man gerade bereist. ${ }^{23}$ Dass es keinen Tag-Nacht-Zyklus gibt, wie man ihn in heutigen Spielen mit offener Welt erwarten würde, mag den technischen Limitationen der Entstehungszeit von SHADOW OF THE COLOSSUS geschuldet sein. Dennoch ist es vielleicht kein Zufall, dass noch dieser Umstand an die antiken Vorstellungen vom Asphodeliengrund gemahnt: „Das Land der Toten dachte man sich nicht nur unter der Erde, sondern es war auch mit dem Westen verbunden“; ${ }^{24}$ also nicht nur mit Nacht und Dunkelheit, sondern, zumindest metonymisch, desgleichen mit der sinkenden Sonne.

Zusammengefasst kann man sagen, dass SHADOW OF THE COLOSSUS die besonderen Möglichkeiten des Mediums Videospiel, die Erkundung der mythopoetischen Welt buchstäblich in die Hand der Spielerinnen und Spieler zu legen, dazu nutzt, eine Zeit des Totenreichs zu gestalten. Der Zauber der gefallenen Welt, die auch hier mit einer Unzahl verborgener Geschichten und Abenteuer lockt, bleibt dabei erhalten. Zugleich jedoch wird er gegen sich selbst gekehrt - man könnte dies den „Autodestruktionen“ hinzufügen, die Thomas Hensel in Bezug auf SHADOW OF THE COLOSSUS ausmacht ${ }^{25}$-, da sich das Verbotene Land weigert, die Versprechen des Fantasy-Modus einzulösen.

Um deutlich zu machen, was das für das Verhältnis von SHADOW OF THE COLOSSUS zu diesem Modus bedeutet, und natürlich auch für die Poetik des Spieles insgesamt, will ich nun auf Wander und die Beziehung zwischen Spieler und Avatar eingehen.

23 Hierin gleicht die Spielwelt von SHADOW OF THE COLOSSUS übrigens jenen der DARK-SOULS-Trilogie. Vgl. das folgende Kapitel dieser Studie.

24 Grant/Hazel: Lexikon der antiken Mythen und Gestalten, S. 177.

25 Vgl. Hensel: Autodestruktionen | Autoikonoklasmen des Computerspiels, S. 103-112. 


\section{Ratloser Triumph}

Es gehört zu den Eigentümlichkeiten von SHADOW OF THE COLOSSUS, dass wir zu Beginn des Spieles ebenso viel über Wander wissen wie am Ende des Abspanns. Mit dem Pakt, den er und Dormin schließen, ist, so scheint es, alles gesagt. Wander ist gleichsam eingeschmolzen in den unbedingten Willen, eine tote junge Frau wieder zum Leben zu erwecken. Das ist der Glutkern der Figur, um den sich kaltes, formloses Gestein anlagert. Anders ausgedrückt: Das Spiel erlaubt uns nicht, eine Perspektive auf Wander einzunehmen, die unabhängig wäre von seiner Entscheidung, jeden Preis zu zahlen, damit Mono ihre Seele wiedererlangt. Mit nur geringer Übertreibung könnte man sagen: Die Figur ist diese Entscheidung, dieser Wille.

Denn was wissen wir sonst von Wander? Ziemlich wenig, fast nichts. Genau genommen wissen wir nicht einmal, in welchem Verhältnis er zu Mono steht. Ist sie seine Geliebte? Seine Schwester? Seine Herrin? Einmal mehr verweigert SHADOw OF THE COLOSSUS die Antworten. Ebenso wenig wissen wir, was Wander mit Lord Emon verbindet, dessen Gestalt die erste Hälfte der langen Schlusssequenz - von der noch zu reden sein wird - beherrscht. Ist er ein Sohn des Lords? Ein Vertrauter? Ein ehemals geschätzter Schüler? Oder war er schon immer der Ausgestoßene und Verachtete, als der er vom Ende des Spiels her erscheint? Auch auf diese Frage gibt uns SHADOW OF THE COLOSSUS keine Antwort. ${ }^{26}$

Für die Spielerinnen und Spieler erscheint Wander also gleichsam als Verkörperung eines zielgerichteten, unbedingten Willens. Hier nun kommt es zu etwas sehr Merkwürdigem. Denn wie positioniert SHADOW OF THE COLOSSUS den Spieler in Hinblick auf diesen Willen?

Konstantin Mitgutsch schreibt: „The motive of the player to fight the unbeatable Colossi, arises from the passion to revive his lost girl.“27 Die Vermischung von Spieler- und Figurenperspektive ist vor allem in der journalistischen Rede über Videospiele durchaus gängig. Im Fall von SHADOW OF THE COLOSSUS scheint sie jedoch paradoxerweise auf ihr Gegenteil zu verweisen: den Umstand nämlich, dass es für die Spielerinnen und Spieler nahezu unmöglich ist, sich in der ästhetischen Erfahrung von SHADOW OF THE COLOSSUS anzuschließen an die Leidenschaft und

26 Die Tatsache, dass Wander und Lord Emon ähnliche Kleidung tragen, scheint dafür zu sprechen, dass die beiden in einem engen Verhältnis zueinander standen; ebenso wie der Umstand, dass Wanders Schwert - so legt das Ende des Spiels nahe - eigentlich dem Lord gehört. Doch letztlich sind das alles Spekulationen.

27 Konstantin Mitgutsch: Passionate Digital Play-Based Learning. (Re)Learning in Computer Games like SHADOW OF THE Colossus. In: Eludamos. 3 (2009), H. 1, S. 9-22, hier: S. 17. 
den Schmerz des Avatars. Mit aller Nachdrücklichkeit wird behauptet, dass Wander ganz durchdrungen ist von diesen Gefühlen; zugleich fallen sie in die Leere, die sich auftut zwischen dem melancholischen Pathos der Eröffnungssequenz und der Ungreifbarkeit der Figuren und ihrer Beziehung zueinander. Diese Ungreifbarkeit betrifft ja auch Mono, über deren Leben und Sterben sich das Spiel völlig ausschweigt. „She was sacrificed, for she has a cursed fate“, sagt Wander. Was das aber bedeuten mag, wird, wenn überhaupt, erst ganz am Ende von SHADOW OF THE COLOSSUS deutlicher.

Es findet also eine Dissoziation statt zwischen Spieler und Avatar. Man wird zum Vollstrecker eines Willens, den man nicht versteht, dem keine eigene Wahrheit entspricht, dem man radikal äußerlich bleibt. Das ist durchaus untypisch für den Fantasy-Modus, der eher danach strebt, die Gefühle der Figuren - ihre Hoffnung und ihre Verzagtheit, ihre Tapferkeit und ihre Angst, ihr Staunen, ihre Liebe, ihren Zorn - mit jenen der Leserinnen, Zuschauer oder Spielerinnen in einem geteilten Resonanzraum zu verbinden. Und auch für Videospiele ist dies eher untypisch; zumal für solche, die dem Genre Fantasy zuzurechnen sind. Natürlich geht es nicht darum, das Spielgefühl mit der mutmaßlichen Innerlichkeit des Avatars kurzzuschließen. Aber wenn eine Figur verzweifelt um ihr Leben kämpft, staunend und bibbernd geheimnisvolle Lande erkundet oder, erfüllt von unbedingter Loyalität, alles riskiert, um einen Kameraden oder eine Gefährtin zu retten, trägt es sicherlich nicht zu einer genussvollen ästhetischen Erfahrung bei, die Spielerinnen und Spieler in einem Außerhalb dieser Gefühle zu positionieren. ${ }^{28}$

Genau das aber tut SHAdow of THE colossus. Nachdem Dormin den Spieler in die Weite des Verbotenen Landes entlassen hat, mag er vor allem eines fühlen: Ratlosigkeit. Von den Kühnheiten, die die Entwickler um Fumito Ueda gewagt haben, ist es vielleicht die größte, dass diese Ratlosigkeit nicht etwa gemildert oder gar aufgehoben wird, wenn Wander in die Schlacht gegen die Kolosse zieht, sondern dass sie umgekehrt gerade hier - wo das Drama in der heroischen Bewährungsprobe, dem archetypischen Kampf von David gegen Goliath, seine höchste Zuspitzung findet - ins Quälende sich steigert.

28 Das gilt natürlich auch für Spiele, die nicht (oder nicht vorwiegend) mit dem FantasyModus arbeiten. THE LAST OF US, um ein zwar berühmtes, jedoch zugegebenermaßen wenig repräsentatives Beispiel zu nennen, könnte offensichtlich nicht funktionieren, wenn sich die Spielerinnen und Spieler abgeschnitten fühlen würden von dem Prozess, in dem Joels väterliche Liebe zu Ellie heranreift und wächst, gegen all seine Widerstände. Denn nur, weil dieser Prozess auch Teil der Spielerfahrung ist, kann vor allem das Finale seine verstörende Wucht entfalten, wenn Joel die Entscheidung trifft, dem Rest der Menschheit lieber die Chance auf ein Heilmittel gegen den Virus des Cordyceps-Pilzes vorzuenthalten, als dem Tod des Mädchens beizustimmen. 
Denn wie stellen sich diese Schlachten dar? Zunächst einmal muss man sagen, dass das Spiel durchaus die affektpoetischen Register wechselt, wenn Wander einen Koloss aufgespürt hat.

Sarah Cameron Loyd Grey beschreibt das wie folgt:

From vast and dead, time suddenly becomes shockingly full. When one's character approaches, the scene zooms in upon the colossus that must be slain. What constitutes the visual field is out of the player's control as she is directed towards this task. Urgent, anticipatory music begins. ${ }^{29}$

Anstelle von Weite und Leere tritt also Fülle, Überfülle gar, sowohl zeitlich als auch räumlich; und die Stille der Suche, noch vertieft durch das Trappeln der Hufe, das Schnauben und Wiehern des Pferdes, das Rauschen des Windes, das Plätschern und Murmeln von Gewässern sowie das Zwitschern von Vögeln, die Stille der Suche also wird ersetzt durch orchestrales, mitunter martialisches Drängen.

Aber wie geht es dann weiter? Ähneln die Kämpfe gegen die Kolosse etwa jenen, die der Spieler zu bestehen hat, wenn er sich an die riesenhaften Endgegner der DARK-SOULS-Trilogie wagt? Auf den ersten Blick könnte man das meinen. Doch tatsächlich offenbart der Vergleich gerade, wie unvergleichlich eigenwillig die Gefechte in SHADOW OF THE COLOSSUS gestaltet sind. Bei den DARK-SOULS-Bossen mag es sich um Ritter, Prinzen, Könige, Hexen, Spinnen, Drachen, Dämonen, Wasserspeier aus lebendem Stein oder noch bizarrere Albtraumgestalten handeln - fast allen ist gemein, dass sie in ihren Angriffen eine furchteinflößende Aggressivität aufweisen. Die mag mal graziös und beinah betörend daherkommen (wie bei der Dancer of the Boreal Valley aus DARK SOULS III), mal Züge des Wahnsinns und der Verzweiflung tragen (wie bei Oceiros, the Consumed King, ebenfalls aus DARK SOULS III) oder sich gelegentlich den Gestaltungsformen der Groteske annähern (wie bei dem Gaping Dragon aus dem ersten Teil); stets jedoch sorgt sie für schweißtreibende, nervenaufreibende und adrenalinpumpende Kämpfe, welche die Spielerinnen und Spieler oft genug an den Rand der Verzweiflung treiben, in Anbetracht der schier unüberwindlichen Macht ihrer Gegner, sodass der schlussendliche Sieg, wenn er denn kommt, umgekehrt aber auch wahre Triumphgefühle auszulösen vermag. ${ }^{30}$

Nichts davon findet sich bei SHADOW OF THE COLOSSUS. Das beginnt damit, dass die Kolosse - „ihres Zeichens anthropomorphe und zoomorphe Mischwesen, die zwar von einem blutähnlichen, schwarzen Lebenssaft durchströmt, aber aus steinernen, gar architektonischen Versatzstücken zusammengesetzt und statt von

29 Grey: Dead Time, S. 236.

$30 \mathrm{Vgl}$. zu der DARK-SOULS-Trilogie das entsprechende Kapitel in dieser Studie. 
einem Fell von saftigem Gras überzogen sind“31 - fast allesamt eine majestätische Schönheit ausstrahlen. Aufgrund ihrer, von Hensel treffend beschriebenen, ${ }^{32}$ hybriden Rätselhaftigkeit hat diese Schönheit zwar etwas Fremdartiges und Wunderliches; zugleich jedoch wirkt sie stets in sich harmonisch, mitunter gar anmutig. Wichtiger noch: Als harmonisch oder organisch kann auch das Verhältnis der Kolosse zu der Spielwelt beschrieben werden. Das wird spätestens deutlich, wenn der Spieler einen seiner Gegner zu Fall gebracht hat.

Dann nämlich

bricht der massive Körper in sich zusammen und geht in das natürliche Tableau der umgebenden Landschaft ein, wird gleichsam terraformiert. Was einst ein Antlitz war, wandelt sich in ein schroffes Steingesicht, was einst ein Körper in eine erodierte Erdmasse, welche in Bodenwellen ausläuft, die nur von Ferne noch an Extremitäten erinnern. ${ }^{33}$

Im Grunde aber ist von Anfang an spürbar, wie sehr die Kolosse dem Verbotenen Land zugehörig sind. Wie beschrieben, muss Wander seine Gegner ja zunächst aufspüren, ehe er gegen sie kämpfen kann. Und die Spielerinnen und Spieler werden es kaum vermeiden können, sich im Verlauf der manchmal langen und mühseligen Suche nach dem nächsten Widersacher wie Eindringlinge zu fühlen. Denn die Kolosse stehen nicht einfach in der Gegend herum und warten darauf, angegriffen zu werden. Sie hausen in Höhlen und Ruinen, in Schluchten und Seen, haben sich eingenistet, fast möchte man sagen: eingeborgen, in das Verbotene Land.

$\mathrm{Zu}$ allem Überfluss erweisen sich diese ebenso seltsamen wie erhabenen Kreaturen oft genug als friedfertig. Viele von ihnen bemerken Wander zunächst gar nicht, wollen einfach nur ihre Kreise ziehen. Und selbst, nachdem man sie angegriffen und verletzt hat, tun manche Kolosse wenig mehr, als dass sie versuchen, den Winzling, der sich da an ihnen festkrallt, abzuschütteln. So verhält es sich etwa bei dem zweiten Gegner, Quadratus, einem Wesen, das an einen Bullen, einen Elefanten oder ein Mammut gemahnt und in einer Höhle am Meeresufer lebt; oder bei dem dreizehnten Koloss, Phalanx, einer wahrlich gigantischen, dabei völlig harmlosen Flugschlange, deren Heimat die Wüste ist.

Christopher Michael Yap hat darauf hingewiesen, dass die langen Zeiten der Stille, der Ruhe und des Alleinseins in dem Verbotenen Land, die mit der Suche nach den Kolossen einhergehen, eigens dafür gemacht scheinen, um den Spielerinnen und Spielern die Gelegenheit zu geben, über ihre Handlungen

31 Hensel: Autodestruktionen | Autoikonoklasmen des Computerspiels, S. 103-104.

32 Wobei es, wenn die Pingeligkeit erlaubt ist, zumindest was den Remaster und das Remake des Spieles betrifft, doch richtiger wäre, von Fell und Gras zu sprechen.

33 Hensel: Autodestruktionen | Autoikonoklasmen des Computerspiels, S. 104. 
nachzudenken. Tue ich das Richtige? - für Yap ist das der Gedanke, der sich immer wieder aufdrängt, die ebenso poetische wie melancholische Leere jener Streifzüge zu füllen. ${ }^{34}$ Er hat völlig recht, geht aber meines Erachtens nicht weit genug. SHADOW OF THE COLOSSUS zielt ja nicht auf eine müßige, irgendwie unbeteiligte Reflexion über moralische Kategorien, sondern will - wie wohl die meisten Kunstwerke - eine ästhetische Erfahrung gestalten, die das ganze Sein beansprucht. Anders gesagt: Die Frage, ob man das Richtige tut, stellt sich mit viszeraler Dringlichkeit, mag zu körperlichem Widerwillen, der an Ekel grenzt, ja zu Wut und Empörung über Wander, über das Spiel, über die eigene Persistenz sich intensivieren, wenn man Stunde um Stunde die immergleichen Abläufe exekutiert, um noch einen, und noch einen Koloss zu jagen und zu töten.

Die Kämpfe selbst sind keineswegs dazu angetan, derartige Unbehaglichkeiten in digitalem Heroismus aufzulösen. Das liegt nicht zuletzt daran, dass noch die Angriffe der Kolosse, die - wie der echsenhafte Kuromori (Nummer acht) oder der Riese Argus (Nummer fünfzehn) - tatsächlich darauf aus sind, Wander ums Leben zu bringen, als verhältnismäßig ungefährlich, weil langsam und vorhersehbar, sich erweisen. Gefragt sind also nicht blitzartige Reflexe und Gamepad-Akrobatik, sondern die richtige Taktik. Die wahre Schwierigkeit besteht in ausnahmslos allen Fällen darin, dass man zunächst herausfinden muss, wo die Kolosse überhaupt verwundbar sind - und wie man ihre verwundbaren Stellen erreicht.

„Was dann kommt“, schreibt Christian Stöcker,

würde in traditionellen Game-Taxonomien als „Plattfomer“ eingestuft: Der Held muss einen Weg hinauf finden, sich am Fell festklammern, an Knochenfortsätzen und Rüstungsteilen entlang hangeln, um irgendwann einen angreifbaren Punkt zu erreichen, in den er sein Schwert stoßen kann. Die Kämpfe mit den Kolossen sind Kämpfe gegen wandelnde Berge, Felsbrocken-Rodeos. ${ }^{35}$

Um diese „Rodeos“ zu bestehen, braucht es zwar Geschicklichkeit und das richtige Timing, vor allem aber Geduld und Beharrlichkeit; Mitgutsch spricht deshalb

34 "As if to further highlight the nigh-complete isolation of the Forbidden Land, travelling to many colossi often takes a relatively long time, during which all the player can do is simply take in the view. In this game, during the Colossus-searching phase, you are quite literally alone with only the thoughts and ponderings you brought with you into the game. The quietude of the game at these times actually serves as an impetus for introspection for the player, and I believe that it is these meditative spaces which allow the player the space to reflect upon their actions and role within the fictional world. Put simply, it allows the player the time and opportunity to ask themselves if what they are doing is right.“ Yap: Reflections on SHADOW OF THE COLOSSUS.

35 Stöcker: Ein stilles Meisterwerk. 
zutreffend von einer „riddle-like challenge“. ${ }^{36}$ Wenn man nicht findig genug ist, um geschwind einen Weg auf die Körper der Kolosse $\mathrm{zu}$ entdecken, deren Schwachstellen zu erkennen und die richtige Kletterroute auszumachen, kann es durchaus geschehen, dass die Kämpfe über viele Minuten hinweg so ablaufen, dass Wander mehr oder weniger ziellos seinen Widersacher umkreist, der seinerseits mehr oder weniger ineffektive Attacken führt. Der wohlige und ehrfurchtsvolle Schauer, welcher die Spielerinnen und Spieler beim ersten Anblick eines Kolosses überkommen mag - sie werden jeweils in kurzen filmischen Sequenzen eingeführt, die alle Register des Kunsterhabenen ziehen -, verkehrt sich dann früher oder später in peinsame Frustration. Da diese zudem mit einer gewissen Ödigkeit einhergeht, sind die Kämpfe in SHADOW OF THE COLOSSUS und in der DARK-SOULS-Reihe, was das betrifft, eher an gegenläufigen Enden der affektpoetischen Skala zu verorten. ${ }^{37}$

Trotz allem wird es irgendwann so weit sein, dass man den Kolossen den Garaus macht. Dann freilich kann von Ödigkeit keine Rede mehr sein. Allerdings auch nicht von beglücktem oder wenigstens erleichtertem Jubel. Den Todesstoß versetzt man seinen Gegnern zumeist, indem man das Schwert in ihre Schädeldecke rammt. Hier stellt das Spiel die hilflose Verwundbarkeit noch des mächtigsten Riesen aus; und wenn einer dieser Riesen schließlich fällt und sich - auf die von Hensel beschriebene Weise - sterbend dem Land angleicht, dem er entstammt, so fällt er quälend langsam, in einer Totalen, die noch einmal seine ganze Majestät zeigt, und sein Ende wird von einem stets sich wiederholenden, ätherischen Klagegesang begleitet.

Vergegenwärtigt man sich all das, kommt man kaum umhin, Christian $\mathrm{Hu}-$ berts beizustimmen, wenn er schreibt: „Jeder Tod eines Kolosses hinterlässt bleibende Wunden.“38 Einen ähnlichen Gedanken formuliert Konstantin Mitgutsch, für den die Kämpfe in SHADOW OF THE COLOSSUS schlichtweg „miserable and ugly“ sind; seine Schlussfolgerung lautet wie folgt: „the victory is not at all glorious, it is rather tragic and breathtaking. It is like defeating someone innocent, who did not even offend you“ - sodass sich Wanders Siege für die Spielerinnen und Spieler also darstellen als „,a strange and hurtful experience“ 39

36 Mitgutsch: Passionate Digital Play-Based Learning, S. 14.

37 Eine Spannung, die mit DARK-SOULs-Bosskämpfen vergleichbar wäre, stellt sich wohl am ehesten im Kampf mit Dirge (der zehnte Koloss) ein. Diesen Kampf kann man nur bestehen, wenn man auf dem Pferd reitet, sich im Sattel umdreht und die Augen der Sandschlange mit Pfeilen spickt, während sie einen in rasendem Tempo verfolgt.

38 Huberts: Raumtemperatur, S. 57.

39 Mitgutsch: Passionate Digital Play-Based Learning, S. 18. Ähnlich heißt es bei Kirsten Rodning: „To a casual observer who is not closely following the story, this might seem like a simple tale of chivalry. A closer interpretation of the events that are taking place, however, would set a player into an uneasy state - it doesn't seem right for the boy to be killing these colossi. Many of 
In der Tat, was für ein sonderbares Spiel ist SHADOw OF THE COLOSSUs! Auf Gedeih und Verderb an einen schweigsamen Jüngling gebunden, über den man nichts weiß, außer dass er um jeden Preis eine tote Frau retten will, über die man ebenfalls nichts weiß, reitet man durch ein Land, das sich dem unerwünschten Eindringling radikal verschließt, und muss Kolosse besiegen, die man lieber in Frieden lassen, denen man es vielleicht sogar mehr als Wander und Mono gönnen würde, ein langes, gesundes Leben zu genießen. So wird die Diskrepanz zwischen der Entschlossenheit des Avatars und der Ratlosigkeit der Spielerinnen und Spieler größer mit jedem Triumph, jedem Gegner, der fällt, weitet sich von einer Kluft zu einem Abgrund, bis zum Ende.

\section{Trauer gegen Trauer}

Wenn das zutrifft, stellt sich eine weitere Frage, die, so meine ich, ins Herz der Poetik von SHADOW OF THE COLOSSUS zielt: Wem oder was, so lautet sie, gilt eigentlich die Melancholie, die das Spiel von den ersten Momenten der Eröffnungssequenz an erfüllt? Wie gesagt: Wanders Schmerz bleibt den Spielerinnen und Spielern ja weitgehend äußerlich; und so dürften nicht wenige unter ihnen geneigt sein, eher um die Kolosse zu trauern als um die geschichts- und identitätslose Polygonleiche Mono. Vielleicht geht es exakt darum. Das jedenfalls legen die gleichermaßen knappen wie hilfreichen Ausführungen von David Thomas nahe. „The game's central feature is that it uniquely presents the game level itself as the level boss“, schreibt er. ${ }^{40}$ Das heißt: „the hero combats the environment

them are docile and do not seem to be causing any harm to anyone." Kirsten Rodning: SHADOw OF THE COLOSSUS as an Example of Post-Modern Literature. In: Video Games as Literature, 7. Februar 2013, http://www.videogamesaslit.com/2013/02/shadow-of-colossus-as-example-of-post. html [letzter Zugriff: 12.05.2020]. Und wirklich: Selbst der letzte Koloss, Malus, der anfangs eine gewisse Boshaftigkeit ausstrahlt, scheint in seinen Handlungen eher von der Neugier getrieben, sich diesen seltsamen Zwerg genauer anzuschauen, wenn Wander ihn erst einmal erklettert hat. 40 David Thomas: SHADOW OF THE Colossus. Player versus Terrain. In: Friedrich von Borries/ Steffen P. Walz/Matthias Böttger (Hg.): Space Time Play. Computer Games, Architecture and Urbanism: the Next Level. Basel 2007, S. 460-461, hier: S. 460. Alexander Lehner, der ebenfalls eine ökokritische Interpretation von SHADOW OF THE COLOSSUS vorlegt, kommt zu ähnlichen Schlussfolgerungen. Er schreibt: „SHADOW OF THE COLOSSUS offers a representational and procedural commentary on how the environment and (as its extension) its inhabitants are treated within a neoliberal mind-set. The colossi are symbolic for the living and non-living parts of the environment as well as for its ludic function as a kind of resource to be gathered. Through a friction between the ludic core and the narrative/representational shell, however, SHADOW OF THE COLOSSUS does not offer a triumphant power fantasy in the spirit of neoliberal logic, but makes the player painfully aware of the consequences of her actions and choices. She is forced 
itself. It's a question of man versus nature, the player versus the environment as represented by the colossi.“" 41

Und Thomas schlussfolgert:

The timeless story of the human environment as a transformation of the natural environment echoes throughout the game. Maneuvering the hero up a colossus like a skilled rock climber scaling a peak, the gamer plays out man's domination over nature. Even though a geyser of blood exploding as the killing blow is struck replaces the traditional flag placed at the mountain summit, the parallels remain obvious and powerful. [...] Ultimately, the game's rendering of our efforts to mindlessly destroy and transform nature, to terraform the land's creatures, the colossi, provides a unique look at how people mark, control and contest terrains, how blood and soil intertwine. And it provides a platform for discussing and experiencing the constant turmoil of a landscape racked by ghostly motivations and demands of the human heart. ${ }^{42}$

Der ökokritische Ansatz, den David Thomas verfolgt, mag SHADOW OF THE COLOSsus nicht in jeder Hinsicht gerecht werden. Eines aber macht er sehr deutlich: dass das Spiel nämlich, insofern es hier um einen Kampf des Menschen gegen die Natur geht, von diesem Kampf nicht einfach erzählt oder ihn zum narrativen Movens macht;

vielmehr tritt der Avatar gegen eine Landschaft an oder anders gesagt: gegen die Spielwelt respektive das Setting selbst. Dieses ist mithin nicht bloße Bühne für das Spielgeschehen, sondern im Gegenteil Antagonist des Spielers, ein handfester Non-Player-Character. ${ }^{43}$

Eine Deutung, für die auch spricht, dass die Kamera in den Kämpfen gegen die Kolosse so unpräzise und träge funktioniert, dass man tatsächlich den Eindruck bekommen könnte, sie arbeite als Agent der Spielwelt aktiv gegen einen Erfolg des Spielers an.

Man kann die Spielwelt von SHADOW OF THE COLOSSUS also als antagonistische Figur begreifen, die sich Wander nicht nur in Gestalt der Kolosse entgegenstellt, sondern sein Streben mit sämtlichen in ihrer Macht stehenden Mitteln zu vereiteln

to realize her own complicity in the destruction of the environment in playing by the rules of the game, which can be connected to the same behavior in the empirical reality. The ending, however, leaves hope for the protagonist and the player, since both are left with the marks of their deeds; the protagonist with his demonic horns and the player with her dreadful feeling of defeat and complicity. “ Alexander Lehner: A Short Theory of Ecocritical Metagames. SHADOW OF THE COLOSSUS and EVERYTHING. In: Paidia. Zeitschrift für Computerspielforschung, Sonderausgabe: Repräsentationen und Funktionen von Umwelt im Computerspiel, 28. Februar 2018, http://www.paidia.de/a-short-theory-of-ecocritical-metagames-shadow-of-the-colossus-and-ever ything/ [letzter Zugriff: 12.05.2020].

41 Thomas: SHADOW OF THE COLOSSUS, S. 461.

42 Thomas: SHADOW OF THE COLOSSUS, S. 461.

43 Hensel: Autodestruktionen | Autoikonoklasmen des Computerspiels, S. 104. 
sucht. Ihre Weite und Leere und Opazität - und vielleicht gar ihre Einflussnahme auf den Programmcode - zählen zu diesen Mitteln. Warum aber tut sie das? Was will sie? Und was sucht sie zu verhindern? Das wird erst vom Ende des Spiels her deutlich.

Schon jetzt aber lässt sich vor diesem Hintergrund die Vermutung äußern, dass die Melancholie, die von den ersten Momenten der Eröffnungssequenz zu spüren ist, gar nicht Wander zugeschrieben werden muss, sondern der Spielwelt selbst. Daraus folgt freilich nicht, dass man sich den Protagonisten von SHADOW OF THE COLOSSUS als herzlosen Betrüger zu denken hätte, der irgendwelche schurkischen Pläne hegt und die Trauer um Mono nur vortäuscht, um wen immer (vielleicht gar den Spieler) hinters Licht zu führen. Vielmehr verhält es sich so, dass SHADOW OF THE COLOSSUS uns zwar von Wanders Trauer erzählt, zugleich jedoch, in der beschriebenen Weise, alles tut, um uns von seiner Trauer abzuspalten und uns stattdessen eine andere Trauer spüren zu lassen. Diese andere Trauer ist gleichsam eine Emanation der Spielwelt: In ihr beweint sie das Verbotene Land - seine sanfte Schönheit, die Fluch und Verderben anheimgefallen ist - und späterhin den Tod der Kolosse.

Die Position, in welche SHADOW OF THE COLOSSUS den Spieler bringt, ist somit noch paradoxer als bislang angenommen: Mit jeder einzelnen Handlung vergrößert er die Traurigkeit, die er, in Gestalt des Helden Wander, doch eigentlich aufheben oder wenigsten mildern sollte; und indem er das tut, zieht ihn die Spielerfahrung unweigerlich auf die Seite, die er im selben Moment bekämpft, wenn er den Avatar in den Kampf gegen einen weiteren Koloss schickt. Damit wird der Spieler sozusagen zum doppelten Verräter: an Wander (dessen Trauer ihn eigentlich nicht schert, obgleich er unter ihrem Banner reitet) und an der Spielwelt (deren Trauer ihn sehr wohl schert, da er sie am eigenen Leib fühlt, die er aber nichtsdestotrotz verschlimmert). SHADOW OF THE COLOSSUS zwingt ihm diese Position zwar nicht gerade auf (im Prinzip wäre es ja möglich, und vielleicht sogar erfreulich, Stunde um Stunde ziellos durch das Verbotene Land zu reiten), lässt ihm allerdings auch keine andere Wahl, als sie einzunehmen, insofern er überhaupt irgendeinen Fortschritt erleben möchte.

In gewisser Weise kämpfen die Spielerinnen und Spieler hier also gegen sich selbst. Zumindest kann man sagen, dass sie sich in der Erfahrung von SHADOW OF THE COLOSSUS als gespalten erleben. Auf den ersten Blick scheint es, als würde die lange Schlusssequenz des Spiels diese Gespaltenheit auf die Spitze treiben, indem sie - ein weiteres Paradoxon - den inneren Widerstand, den die Spielerinnen und Spieler gegen ihr eigenes Tun empfinden, in einer allumfassenden Negation auflöst. 


\section{Die Aufhebung der Spaltung}

Nachdem Wander den zwölften Koloss, das fremdartige Wasserwesen Pelagia, bezwungen hat, ereignet sich etwas Unerwartetes. Zu diesem Zeitpunkt werden die meisten Spielerinnen und Spieler schon eine Reihe von Stunden im Verbotenen Land zugebracht haben. Sie haben den immergleichen Ablauf von Suche und Kampf unterdessen so oft vollzogen, dass sich vielleicht die Empfindung eingestellt hat, die monumentale Erhabenheit der Ruinen, Kolosse und Landschaften sei um einen Schlund gähnender Leere angelagert. Jetzt aber unterbricht etwas die gewohnte Bilderfolge: Wie immer wird Wander nach dem Niedergang des Kolosses von schwarzem, tentakelartigem Rauch durchdrungen oder durchbohrt; wie immer führt das zu einer Ohnmacht, die (vor allem wenn er, wie jetzt, reglos im Wasser treibt) sehr viel Ähnlichkeit mit einem Tod hat. Dies nicht zuletzt darum, weil im Anschluss, wie immer, eine Szene folgt, in der etwas oder jemand (Wanders Seele?) durch einen Tunnel der Schwärze auf ein gleißendes Licht zufliegt.

Doch wenn das Licht hell aufgeglommen ist, die ganze Bildfläche erfüllt hat, finden wir uns diesmal nicht mit Wander im Tempel wieder, sondern sehen zunächst einen Trupp Reiter, der durch eine Landschaft prescht, die jener gleicht, welche wir in der Eröffnungssequenz erblickten. Die Reiter halten, ihr Anführer ein Mann, der eine Eulenmaske trägt, wie sie zu Anfang des Spieles mit dem rätselhaften Erzähler assoziiert wurde ${ }^{44}$ - murmelt, es sei jetzt nicht mehr weit, und der Trupp setzt den eiligen Ritt fort: in Richtung von Dormins Tempel, der in der Ferne aufragt.

Ganz am Ende, wenn der letzte Koloss gefallen ist, begegnen uns diese Reiter dann wieder. Zu Beginn der 25-minütigen Schlusssequenz galoppieren sie über die Brücke, die Wander am Anfang des Spiels passiert hat. Auch für sie hebt sich das Steintor. Die maskierten Reiter ziehen Schwerter und geleiten ihren Anführer, den sie als „Lord Emon“ ansprechen, hinab in die Tiefe des Tempels - gerade rechtzeitig um zu sehen, wie in dem verbliebenen Idol ein gleißendes Licht aufscheint, das es bald von Innen her zersprengt.

Während der Lord, nun mit geöffneter Maske, eine Art Ritual an der toten Mono vollzieht, vielleicht Gebete spricht, erscheint auch Wander im Tempel. Einmal mehr kommt sein lebloser Körper auf dem Steinboden zu liegen. Lord Emon stellt ihn, der mühsam, gequält und offenbar am Ende seiner Kraft sich aufrappelt,

44 Da der Anführer des Reitertrupps und der Erzähler mit derselben Stimme sprechen, ist jener wohl in der Tat mit diesem identisch. 
zur Rede: Ob er, Wander, wisse, was er getan habe? Nicht nur habe er das Schwert, eben „the Ancient Sword“, gestohlen und ohne Erlaubnis dieses verfluchte Land betreten, sondern obendrein einen verbotenen Zauber angewendet. Dann offenbart der Lord, dass Wander die ganze Zeit über nur benutzt worden sei, und gibt seinen Männern den Befehl ihn zu töten: „Eradicate the source of the evil“ - es sei besser, Wander von seinem Elend zu erlösen, als zu erlauben, dass er in diesem Zustand weiterexistiere.

Ein Armbrustbolzen bohrt sich in das Bein des Wehrlosen, der nicht einmal mehr sprechen, geschweige denn kämpfen kann und stöhnend zu Boden fällt. Dann tritt einer von Lord Emons Männern an Wander heran, um ihn mit dem Schwert zu durchbohren.

Doch Wander stirbt nicht. Aus der Wunde, die ihm die Klinge geschlagen hat, sprudelt das schwarze Blut, das auch die Kolosse durchpulste. Noch einmal steht Wander auf, reißt sich das Schwert heraus, streckt die Hand nach der toten Mono aus - und wird dann von der Schwärze, die ihm entströmt, in einen jener Schatten verwandelt, die er zu Beginn mit der Magie des Schwertes vertrieben hat, ehe er erneut zusammenbricht. Als er sich nun zum letzten Mal aufrappelt, ist er nicht mehr er selbst. Wander verändert seine Gestalt, an Spinnenbeine gemahnende Auswüchse treiben aus seinem Rücken hervor, während er anschwillt, wächst und wächst, schließlich als schwarzer, felliger, mit Hörnern und Klauen bewehrter Riese dasteht.

Thomas Hensel beschreibt diesen Prozess als eine „Entanthropomorphisierung“, in deren Verlauf sich Wander

in jenen metaphysischen gottgleichen Dämon verwandelt, genius malignus des Spiels, dessen Stimme ihn die ganze Zeit über geleitet hatte. Es offenbart sich, dass der einzige Zweck der Kolosse Tötung es war, den Dämon aus deren vereinten Essenzen wiederauferstehen zu lassen. Doch der Größen- und Machtzuwachs ist von beschränkter Dauer - alsbald verflüchtigt sich mit jener schwarz-rauchigen Essenz der kolossal gewordene Körper des Avatars, der Spieler verliert den Zugriff auf seinen Stellvertreter - der sich zuverlässig nicht mehr steuern lässt -, bis der Dämonenkörper schließlich selbst auf menschliches Maß schrumpft [...]..$^{45}$

Dieses Schrumpfen ereignet sich, nachdem Lord Emon mit seinen Männern - tatsächlich haben die Spielerinnen und Spieler während der kurzen Zeit, in der sie Dormin steuern, die Möglichkeit, mit der riesigen Faust des Dämons dreinzuschlagen - aus der Tempelhalle geflohen und die schier endlose Treppe empor gelaufen ist. Der Lord, womöglich ein Nachfahr derer, die Dormin einstmals in

45 Hensel: Autodestruktionen | Autoikonoklasmen des Computerspiels, S. 106-107. 
die Steinidole sperrten, versiegelt nun den Schrein, indem er das „Ancient Sword“ in das Wasserbecken am Fuß der Treppe schleudert. ${ }^{46}$ Darauf bricht eine turmhohe Säule blauweißen Lichts aus dem Wasser hervor, die zugleich einen Sog entfaltet, der den verzweifelt sich wehrenden Dormin unerbittlich anzieht, ihn kleiner und kleiner werden lässt, bis es am Schluss wieder Wander ist (was uns nicht zuletzt die ächzenden Laute bestätigen, die dieser mit wohlbekannter Stimme ausstößt), der ins Becken fällt und in dem Gemisch aus Licht und Wasser verschwindet.

Unterdessen überqueren Lord Emon und seine Männer in wildem Galopp die Brücke, die, ähnlich den steinernen Idolen von Licht gesprengt, hinter ihnen einstürzt. Noch ist unklar, ob die Reiter es schaffen werden, das Verbotene Land bei lebendigem Leib zu verlassen. Doch ehe sich ihr Schicksal entscheidet, finden wir uns im Tempel wieder.

In einer Reihe von langen, ruhigen Einstellungen nähert sich die Kamera Mono, betrachtet sie von verschiedenen Seiten, lässt den Blick gleichsam an ihr entlang gleiten, mit deren Haaren und Gewand der sanfte Wind spielt. Etwas Zärtliches, beinah Liebkosendes liegt darin, wie die tote Frau hier beäugt wird. Zugleich suggerieren die verschiedenen Blickwinkel, die immer neuen Annäherungen, eine gewisse Neugierde; als würde der Steinaltar von unsichtbaren Wesen umschwebt, die sich jetzt, da alle Störenfriede - Wander, Dormin, Lord Emon und seine Männer - verschwunden sind, doch einmal anschauen wollen, was ihnen da sozusagen ins Haus gebracht worden ist. Während diese Annäherungen sich vollziehen, werden musikalische Motive der Eingangssequenz aufgegriffen; schon der Klang der Flöten und Streicher erweckt solcherart eine Ahnung, dass sich hier ein Kreis schließen mag. So überrascht es nicht, dass Mono tatsächlich wieder zum Leben erwacht. Damit, wie sie die Augen öffnet, sich aufsetzt und um sich schaut - weder erstaunt noch aufgeregt, vielleicht ein bisschen traurig, vielleicht auch ihrerseits neugierig -, endet eine affektpoetische Bewegung, die den ersten, vom Spiel freilich nicht als solchen markierten Teil der Schlusssequenz umfasst hat.

Halten wir also einen Moment inne. Was geschieht hier? Und wie verhält sich das Finale zu den Stunden davor, dem endlosen Suchen und Kämpfen, dem Schmerz des Spielers am eigenen Tun?

46 Dormin macht Lord Emon persönlich dafür verantwortlich, dass sein Körper in sechzehn Teile zerstückelt worden ist; andererseits spricht er davon, er sei „an eternity“ eingesperrt gewesen, sodass unklar ist, ob es sich bei Emon um den alten Widersacher des Dämons oder einen Nachfahr desselben handelt. Auch in Anbetracht des Alters der Ruinen, die das Verbotene Land überziehen, scheint letzteres weit wahrscheinlicher. 
Hensel deutet dieses Ende so, dass SHADOW OF THE COLOSSUS mit ihm „seine eigene Destruktion in Szene“ setze: „De facto nämlich ist es nicht nur die Spielwelt, die attackiert wird, sondern komplementär auch der Avatar selbst, dem in letzter Konsequenz alles genommen wird, wodurch er sich auszeichnet. “47 Bezogen auf den Gegenstand der vorliegenden Studie kann man ergänzend sagen, dass obendrein der Fantasy-Modus attackiert und destruiert zu werden scheint. Denn was hier geschieht, nimmt sich - wenigstens auf den ersten Blick - wie eine bewusste Verkehrung, ja geradezu wie eine Verhöhnung von Tolkiens Konzeption aus. Anstelle der Eukastrophe tritt eine bittere Katastrophe: Wander wurde betrogen und ausgenutzt, hat sich zum Büttel des Dämons machen lassen, und nur durch Lord Emons beherztes Eingreifen wird der vollständige Triumph des Bösen verhindert.

Bezogen auf Wander, die Mühen des Spielers und den Tod der Kolosse muss man also sagen: Es war alles umsonst. Vor diesem Hintergrund meint man auch zu verstehen, warum die Spielwelt den Avatar als Eindringling behandelte und die ganze Zeit über Trauer trug. In der Tat trauerte sie um sich selbst, da sie dazu verdammt war, die gespaltene Essenz des Dämons in sich zu bergen; und in der Tat trauerte sie auch um die Kolosse, die zwar irgendwie Teile von Dormin waren, zugleich jedoch auch seine Wächter, folglich die Beschützer des Landes, die eine Wiederkehr des Bösen verhüteten - als könnte das, was in seiner Gesamtheit nur vernichtende Schwärze ist, in seinen einzelnen Fragmenten das Gegenteil seiner Selbst anrühren.

Das alles weiß man natürlich nicht. Nichts davon wird einem erklärt oder dargelegt. Vielmehr handelt es sich um ein Gefühl für die Welt von SHADOW oF THE CoLOSSUS, das aus dem Ganzen der Spielerfahrung erwächst. Gerade darum, weil es hier um Erfahrungsqualitäten geht, drängt sich aber auch der Verdacht auf, dass es gar so einfach nicht sein kann. Und zwar schon vor Monos Erwachen, das dem Eindruck der Vergeblichkeit die eigene Faktizität machtvoll entgegenstellt. Denn schließlich hat Wander am Ende ja doch erreicht, was er wollte. War er nicht stets bereit, jeden Preis zu zahlen, um Mono zu retten?

Aber geschieht eigentlich mit Wander im Verlauf der Schlusssequenz nicht auf der Ebene seiner „Entanthropomorphisierung“, sondern was das Verhältnis zu den Spielerinnen und Spielern betrifft? Eigentlich vollendet sich nur, was schon vorher geschehen war. Wer genau hinsah, konnte es bereits recht früh bemerken: Wander fing an, sich zu verändern. Sein Gesicht wurde fahler, feine, schwarze Adern begannen, seine Haut zu durchziehen. Doch erst in der

47 Hensel: Autodestruktionen | Autoikonoklasmen des Computerspiels, S. 106. 
Schlusssequenz, wenn Wander plötzlich Hörner trägt und seine Augen jenen der Kolosse ähneln, wird seine Wandlung unübersehbar. ${ }^{48}$

Wichtig ist nun, dass diese Veränderung keine rein äußerliche ist, sie betrifft die ganze Inszenierung Wanders. Auf einmal spürt man seine Erschöpfung, seinen Schmerz, sein ohnmächtiges Sich-Aufbäumen, seine verzweifelte Liebe. Und plötzlich wird er in der Spielerfahrung zu dem, was er bislang nur qua seiner Funktion als Avatar war: ein Held. Ein tragischer Held, freilich. Ein Held, der sich des Hochmuts und der Verblendung schuldig gemacht hat und der - so scheint es jedenfalls, wenn er von Dormin übernommen wird -, das Gute erstrebend, dem Bösen den Weg bereitete.

Vieles ist buchstäblich ver-kehrt bei diesem Orpheus. Es ist nicht so, dass die Natur ihm verzückt lauscht, dass alle Geschöpfe ihm folgen, dass sogar Bäume und Steine zu ihm kommen. ${ }^{49}$ Vielmehr kann er nicht anders, als die Natur zu peinigen, alle ihre Geschöpfe und sie selbst - in Gestalt der Spielwelt - zum Feind sich zu machen. Und am Schluss ist er es dann, der ein und für alle Mal in die Unterwelt eingeht, mit Leib und Seele sozusagen, indem er sich ihr angleicht.

Doch indem dies geschieht, wird es auf einmal möglich, jenes Mitleid für Wander zu empfinden, das zuvor den Kolossen vorbehalten war. Die Trauer der Spielwelt und die Trauer des Avatars werden im Wahrnehmen und Empfinden des Spielers miteinander versöhnt. Die Spaltung, die ihn während der gesamten Dauer von SHADOW OF THE COLOSSUS mit sich selbst entzweite, wird nun aufgehoben. Bezogen auf Wander ist das ganz buchstäblich zu verstehen, denn in seiner neuen, monströsen Gestalt wird er, wie beschrieben, Teil des Verbotenen Landes, durchläuft eine Anverwandlung nicht nur an Dormin, sondern auch an die Kolosse.

Es ist, als sei diese Aufhebung zugleich die Voraussetzung dafür, dass Mono erwacht. Denn dies geschieht ja, nachdem Dormin, Wander und Lord Emon längst schon verschwunden sind. So bleibt nur die Spielwelt selbst als Agentin dieses Geschehens. Ihr mögen die neugierigen und zartfühlenden Blicke zuzuschreiben sein, mit denen nun die Annäherung an Mono sich vollzieht. Als wäre es jetzt, da Wander den Preis bezahlt hat, endlich möglich, die junge Frau wirklich zu sehen - und auch hierin vereinigen sich nun die Perspektiven der Spielwelt und des Spielers.

48 Das gilt zumindest für das PS4-Remake; in der ursprünglichen Fassung des Spieles war die Veränderung des Avatars schon lange vor dem Ende deutlich auszumachen, was zum Beispiel auch den Verschleiß seiner Kleidung betraf.

49 Vgl. Grant/Hazel: Lexikon der antiken Mythen und Gestalten, S. 314. 
Wenn das aber so ist, kann SHADOW OF THE COLOSSUS nicht in hoffnungsloser Bitterkeit enden. Und tatsächlich eröffnet der zweite Teil der Schlusssequenz noch einmal eine neue Perspektive auf das Geschehen.

\section{Die Eukatastrophe in der Katastrophe}

Was an äußerer Handlung bleibt, ist schnell erzählt. Nachdem sich Mono vom Altar erhoben und umgesehen hat, kommt Agro zu ihr, Wanders schwarzes Pferd, das vermeintlich auf dem Weg zum sechzehnten und letzten Koloss in den Tod stürzte; das Tier hat sich am linken Hinterlauf verletzt, kann sich aber humpelnd fortbewegen. Derweil hält Lord Emon, der es mitsamt seinen Männern auf die andere Seite geschafft hat, eine kurze Rede, die sich wie ein Epitaph ausnimmt - ob auf Wander oder Mono, bleibt unklar. Dann beginnen Mono und Agro den langen Aufstieg zum Dach des Tempels. In dem nun leeren Wasserbecken finden sie einen gehörnten Säugling, bei dem es sich anscheinend um Wander handelt. Mono hebt das Kind auf, hält es in den Armen, betrachtet es einen langen Moment - erstaunt scheint sie noch immer nicht -, folgt dann dem Pferd, das schon begonnen hat, die Stufen zu erklimmen. Irgendwann erreichen die beiden das Steintor, das nach der Flucht von Lord Emon offengeblieben ist. Zielstrebig wählt Agro einen Weg, der weiter nach oben führt, jetzt an der Außenseite des Tempels entlang, und wieder folgt Mono, die immer noch den Säugling in den Armen trägt.

Es erweist sich, dass auf dem Tempeldach, von Säulen, Mauern und steinernen Überresten umgeben, ein blühender Garten wächst. Agro beginnt zu grasen, Mono tritt an das Pferd heran, und ein äsendes Reh, das die beiden zunächst aus einiger Entfernung beobachtet, gesellt sich zu ihnen. Auch ein Eichhörnchen, Tauben und - vermutlich - der uns bekannte Adler kommen herbei. Einen Augenblick scheinen die Tiere das Kind in Monos Armen zu betrachten, dann flattern die Vögel wie auf ein geheimes Signal davon. SHADOW OF THE COLOSSUS endet damit, dass der Adler sich hoch in die Lüfte schwingt, dann auf die Kamera zufliegt, die sich gemeinsam mit ihm vom Tempel entfernt, bald jedoch umschwenkt, sodass sie ihn kurz von unten und dann von hinten betrachtet, während er den Grenzen des Verbotenen Landes zustrebt und durch Nebel und Dunkelheit in einen Nachthimmel einfliegt, der jenem gleicht, den wir ganz zu Beginn des Spieles sahen. Hinter sich ballenden Wolken steht ein voller Mond, und mit diesem Bild werden wir entlassen.

Was fängt man mit einem solchen Ende an? Zunächst einmal scheint es mir wichtig, wie Thomas Hensel zu betonen, dass die Schlusssequenz zwar lang und ereignisreich ist, ,in narratologischer Perspektive allerdings jedes hermeneutische 
Bestreben zum Scheitern verdammt“. ${ }^{50}$ Demgemäß sind die wissenschaftlichen Auseinandersetzungen mit SHADOW OF THE COLOSSUS von einer gewissen Zurückhaltung gekennzeichnet, was die Interpretation des Endes betrifft. ${ }^{51}$ Auffällig ist jedoch, dass häufig die Düsternis und Bitterkeit der Schlusssequenz betont wird, sodass ein Leser, der keine eigene Erfahrung mit dem Spiel hat, zu dem Schluss kommen könnte, der letzte Vorhang falle, nachdem Wander unter die Kontrolle des Dämons gezwungen wurde. ${ }^{52}$

Eine Ausnahme stellt zum einen Hensel selbst dar, der nicht zu erwähnen vergisst, dass es Wander gewährt wird, „am Ende als hörnerbewehrter Säugling in einer Art Garten Eden einen neuen Lebenszyklus zu beginnen“. 53

Und bei Sarah Cameron Loyd Grey heißt es:

At the very end of the story, the character's lover awakens to find the broken and struggling horse returning to her side. She wanders about the ruins and comes upon a grassy knoll that has the first signs of new life. Small creatures hop about cautiously and greenery grows anew. This rebirth is not just a pandering happy ending, since the gamer does not herself overcome all obstacles. Instead, it can be read as a new vista of potential opening up before the gamer as she replays. ${ }^{54}$

50 Hensel: Autodestruktionen | Autoikonoklasmen des Computerspiels, S. 108.

51 Die Spielerinnen und Spieler selbst sind da naturgemäß weniger zurückhaltend. Im Netz finden sich allerlei Theorien, die das Ende von SHADOW OF THE CoLOSSUS erklären sollen. Für den gegebenen Zusammenhang ist die Frage nach der Validität dieser Theorien - sie zielen letztlich auf eine Erklärung, was es mit Monos „cursed fate“ auf sich haben mag - indessen nebensächlich.

52 Es seien einige Beispiele für diese Interpretation zitiert: „In SHADOW OF THE COLOSSUS, the player has the task of slaying giant colossi in order to bring back a loved one from the dead. In the process, the hero starts an inevitable deterioration towards turning into a monster himself.“ Aki Järvinen: Understanding Video Games as Emotional Experiences. In: Mark J. P. Wolf/Bernard Perron (Hg.): The Video Game Theory Reader 2. New York/London 2009, S. 85-108, hier: S. 107.

„SHADOW OF THE COLOSSUS provides a different take on complicity: in this case, we are told that in order to save the girl Mono, we have to kill a series of colossi. These colossi turn out to be large, slow-moving, melancholy creatures that inspire pity rather than fear. Although the girl is eventually saved, it becomes clear during the game that the colossi are actually innocent creatures, and the protagonist is eventually punished for having killed them." Jesper Juul: The Art of Failure. An Essay on the Pain of Playing Video Games. Cambridge/London 2013, S. 109-110.

„And SHADOW OF THE COLOSSUS culminates in the realization that the protagonist has been working in the service of a demon; the player spends the protagonist's final moments controlling a futile struggle against a vortex meant to imprison the demon that has possessed him.“ Jason Tocci: You Are Dead. Continue? Conflicts and Complements in Game Rules and Fiction. In: Eludamos. 2 (2008), H. 2, S. 180-201, hier: S. 198.

53 Hensel: Autodestruktionen | Autoikonoklasmen des Computerspiels, S. 107.

54 Grey: Dead Time, S. 239. Ähnlich betont Alexander Lehner das utopische Potential des Endes von SHADOW OF THE CoLOSSUS. Vgl. Lehner: A Short Theory of Ecocritical Metagames. 
Beide, Hensel und Grey, betonen in ihren Ausführungen das „Neue“. Damit ist meines Erachtens das entscheidende Stichwort gegeben, womit ein Verständnis des Endes von SHADOW OF THE COLOSSUS in seiner Erfahrungsdimension - das heißt, diesseits und jenseits aller Interpretation - möglich wird.

Das Neue beginnt, als Agro auf Mono zuhumpelt, und die junge Frau dem Pferd einen Schritt entgegengeht, um es zu streicheln und zu liebkosen. In diesem Moment findet in SHADOW OF THE COLOSSUS die erste und einzige zärtliche Berührung zwischen zwei Wesen statt, die, wenn man so sagen kann, unterschiedlichen Seinsordnungen angehören. Es ist wohl kein Zufall, dass diese zärtliche Berührung eine andere spiegelt, die sich ereignet, nachdem Wander den achten Koloss, Kuromori, besiegt hat. Da scheint es einen Augenblick lang, als wäre Mono - eine in gleißendes Gegenlicht gehüllte Vision - bereits wieder zum Leben erwacht, und nachdem Wander aus seiner Ohnmacht erstanden ist, streicht er der toten Frau mit dem Handrücken sanft über die Wange. Jetzt am Ende aber ist es die Berührung zweier lebendiger Wesen, die aufeinander zugehen, was in Monos Fall zudem bedeutet, dass sie einen Streifen durch die Säulenbögen einfallenden Sonnenlichts verlässt und sich in den Schatten begibt, um Agro nah zu sein.

Tatsächlich ist in dieser kleinen Berührung eine Gegenlogik entworfen zu dem Gesetz, das die Welt von SHADOW OF THE COLOSSUS bislang beherrschte - ein Gesetz, welches eben vorschreibt, dass allen Handlungen, die der Mensch gegenüber dem Land und seinen Geschöpfen vollzieht, etwas Gewaltsames und Missbräuchliches anhaftet. Was immer Wander gewollt oder gewünscht haben mag, sein Tun mündete stets in Akte der Unterwerfung; und die Kolosse wussten darauf entweder gar nicht oder ihrerseits nur mit zerstörerischer Gewalt zu antworten. Selbst Agro war von dieser Logik nicht ausgenommen. Denn so sehr man die Freundschaft und Treue zwischen Pferd und Reiter spürt, so deutlich ist auch, dass Wander das Tier immer wieder zwingt, dem eigenen Streben zuwider zu handeln: bis zu jenem Sprung über eine zerfallende Brücke, der Agros Sturz und Verletzung zur Folge hat. ${ }^{55}$

55 Vor diesem Hintergrund kommt es mir etwas harmonisierend vor, wenn Mary Flanagan und Helen Nissenbaum schreiben: „The playable character, Wander, develops a deep relationship with his guide horse, Agro. The horse's behavior, however, is programmed, so she does not always respond to commands. Players therefore cannot, drive' the horse as they would drive a car that reacts precisely to their movements. The player must ride the horse in a two-way relationship that is governed by give and take. This control style leads the player to adopt a cooperative mindset. Companionship and collaboration are values inherent in the Wander/Agro relationship.“ Mary Flanagan/Helen Nissenbaum: Values at Play in Digital Games. Cambridge/London 2014, S. 105. 
Die zärtliche Geste bricht somit nicht nur das Weltgesetz auf, dem SHADOw OF THE COLOSSUS bis hierhin unterworfen war (da ist es angemessen, von einer sich verflüssigenden Starre im Sinne Brittnachers zu sprechen), sondern inauguriert zudem eine neue Zeitlichkeit, die der zyklischen Zeit des Totenreichs radikal entgegensteht: War jene durch die immer gleichen Abläufe gekennzeichnet, die von Mal zu Mal stumpfer, leerer, quälender und trübsinniger wurden, entstehen nun Möglichkeiten und Potenzialitäten, eröffnet sich Raum für das Neue.

Die Inszenierung von SHADOW OF THE COLOSSUS unterstreicht die Bedeutung dieser Berührung. Denn noch während sie sich ereignet, schwenkt die Kamera nach oben und in einer Weißblende beginnt der Abspann; im Rahmen der Paratexte eines Spieles oder Filmes wohl die eindeutigste Markierung, dass etwas an sein Ende gekommen ist. Der Abspann stellt sich zunächst dar als Requiem auf die Kolosse, die in ruhigen Einstellungen verabschiedet werden, welche an den Ort der Kämpfe zurückkehren und die zumeist von Sand und Erde bedeckten, teilweise auch schon wieder mit Gras und Gestrüpp, Sträuchern, Büschen und Bäumen bewachsenen Überreste jener gewaltigen Wesen zeigen.

Nachdem das Spiel der sechzehn Kolosse gedacht hat, kehrt es, wie gesagt, zu Lord Emon zurück. Und hier wiederholt sich der Eindruck, dass ein Ende erreicht ist, in dem etwas Neues sich auftut.

„Poor ungodly soul“, sagt der Lord, über die weite Kluft, wo noch vor wenigen Momenten die Brücke stand, auf den Tempel Dormins zurückblickend. „Now, no man shall ever trespass upon this place again. Should you be alive, if it's even possible to continue to exist in these sealed lands ... One day, perhaps you will make atonement for what you've done.“

Das strenge, feierliche Pathos der kurzen Ansprache erscheint seltsam hohl und verfehlt. Vielleicht wird daran greifbar, dass Lord Emon nicht einfach die Macht des Guten verkörpert, sondern ebenfalls eingeschlossen ist in jene Logik von stets sich erneuernder Gewalt und Unterwerfung, die Wander und zweifellos auch Dormin erfasst hat. Fest steht, dass er (ganz buchstäblich) keinen Zugang hat zu dem Neuen, das eben nicht in der Zukunft, sondern hier und jetzt stattfindet - weshalb Reue und Wiedergutmachung etwas sind, das die Gegenwart bereits zu erfüllen beginnt. In diesem Zusammenhang sollte nicht unerwähnt bleiben, dass das Ensemble, das sich im Garten auf dem Tempeldach um den gehörnten Säugling einfindet, mit Mono, Reh und Agro (die in den Paratexten des Spiels stets als Stute angesprochen wird) ein rein Weibliches ist. Als hätte Wander, jeglicher Handlungsmacht entkleidet und auf liebevolle Zuwendung angewiesen, jetzt mehrere Mütter, die, verschiedenen Welten entstammend, sich zusammenfinden in der Sorge um ihn. Dazu passt es, dass das Glück, das in diesem Ende als Möglichkeit und Ahnung aufscheinen mag, eben kein Erstrebtes und Erkämpftes ist, sondern ein Geschenktes - gerade für die 
Spielerinnen und Spieler, die ja die längste Zeit schon keinen Einfluss mehr auf das Geschehen haben. ${ }^{56}$

All das soll keine Interpretation des Endes von SHADOW OF THE COLOSSUS darstellen. Weder wenn man der orpheischen Spur folgt, noch wenn man den Fantasy-Modus als Deutungsmatrix anlegt, kommt man hier zum Ziel. Keine Interpretation also. Wohl aber der Versuch, das Ende des Spiels in seiner Erfahrungsdimension greifbar zu machen. Aus dieser Perspektive betrachtet, kann man sagen, dass die Inszenierung Fumito Uedas weit davon entfernt ist, die Tolkiensche Poetik zu negieren. Im Gegenteil. Es gibt kaum ein Spiel, einen Film, ein Buch, die eine derart vollendete Eukatastrophe aufweisen wie SHADOW OF THE COLOSSUS. Vollendet ist sie gerade in ihrem Überraschenden und Unerwarteten - darin dass sie wahrhaft gegen jede Hoffnung, jede Wahrscheinlichkeit, ja sogar gegen jede Plausibilität und rationale Erklärbarkeit sich ereignet.

Die Art, wie das Gute hier seine eigene Unmöglichkeit anerkennt und im selben Moment überwindet, mag darauf hindeuten, dass es eine Wirklichkeit gibt, die in unserer Wirklichkeit wirkt, dabei jedoch ihren eigenen, unserem Verständnis entzogenen Regeln folgt. Jedenfalls sind die Spielerinnen und Spieler am Ende von SHADOW OF THE COLOSSUS mit der Spielwelt vereint in der Sehnsucht nach dem ganz Anderen des Asphodeliengrunds, dem Jenseits des Totenreichs.

56 Wie bereits zitiert schreibt Sarah Cameron Loyd Grey: „This rebirth is not just a pandering happy ending, since the gamer does not herself overcome all obstacles." Vgl. Cameron Loyd Grey: Dead Time, S. 239. 
